\title{
Charge transport and transfer phenomena involving conjugated acenes and heteroacenes
}

\author{
ANUP PRAMANIK ${ }^{1}$, SANTU BISWAS ${ }^{1}$, SOUGATA PAL ${ }^{2}$ and PRANAB SARKAR ${ }^{1, *}$ (D \\ ${ }^{1}$ Department of Chemistry, Visva-Bharati University, Santiniketan 731 235, India \\ ${ }^{2}$ Department of Chemistry, University of Gour Banga, Malda 732103, India \\ *Author for correspondence (pranab.sarkar@visva-bharati.ac.in)
}

MS received 4 October 2018; accepted 31 October 2018; published online 25 April 2019

\begin{abstract}
Conjugated acenes are very promising for their applicability in different optoelectronic devices. In particular, pentacene (PCn) shows some distinct behaviours, namely high optical absorption and remarkably high carrier mobility which makes it as a landmark semiconductor for use in field-effect transistors or hole transporting materials in organic photovoltaics. During the last few decades, a large number of theoretical and experimental researches has been performed showing the practical applicability of different acenes and heteroatom-doped acenes (heteroacenes). Few reviews are also made in this regard. However, correlating the molecular properties arising from their intrinsic electronic structures with their charge transfer (CT) and transport characteristics is really scattered. Furthermore, very recent understandings on the nanojunctions made of acenes along with suitable electrodes need to be reviewed for further development of their performances in electronic devices. At the same time, the photovoltaic applicability of acenes and heteroacenes has recently been shown as an interplay of the dynamics of CT states as well as the intrinsic charge separation within the molecules or composites. The present review aims to point out those recent observations so as to draw more attention for further development in the area of aceneand heteroacene-based optoelectronic devices. We concentrate more on the structure-property relationships which could guide the device performance. As the subject area is so vast, we put emphasis on the very recent studies on PCn and some lower S,N-heteroacenes.
\end{abstract}

Keywords. Acenes and heteroacenes; optoelectronics; transport properties; photovoltaic activities.

\section{Introduction}

Over the last few decades, $\pi$-conjugated small organic molecules have drawn tremendous attention for their use in different optoelectronic devices such as logic gates, fieldeffect transistors (FETs), memory storage devices, organic photovoltaic cells (PVCs), etc. [1-7]. The solution processability and tunable electronic structures of these conjugated organic molecules make them attractive for such applications [4-7]. The tunable electronic structures of those molecules could easily be realized by functionalizing their aromatic rings by different electron donating or withdrawing groups. Thus, with the help of sophisticated organic synthetic protocols, those molecules are implemented in molecular or organic electronics as per the requirement. Acenes are such a class of $\pi$-conjugated molecules which can be considered as one-dimensional (1D) strips of graphene and they have been identified as potential materials to be used in the next generation of electronic devices [8,9]. One of the most attractive features of acenes is their exceptionally narrow band-gap, which leads to a higher conductivity than that of other similar organic molecules. Pentacene (PCn) is the most popular example amongst the acene family which is supposed to be a benchmark semiconductor in organic electronics [10,11].
This is mainly due to its high field-effect mobility and at the same time, it has also some unique features like high carrier mobility, high amenability to thin film formation, etc. [12-17]. Single molecular contacts using PCn or heteroatomdoped PCn are an interesting issue in this regard and hence, PCn-based molecular junctions using various metallic contacts have been widespread studied theoretically as well as experimentally [11,13,18-21].

$\mathrm{PCn}-\mathrm{Au}$ nanojunctions are studied for various electronics measurements [21-24]. However, during the last couple of years, carbon-based materials, such as carbon nanotubes (CNTs), graphene, etc. are also employed for such junction studies. Even, these materials are sometimes proved to be better than metal contacts because they are directly synthesized at the contact region by using a chemical vapour deposition method and can be patterned by lithographic technique [25-28]. Furthermore, recent advancements in material growth and designing techniques have made two-dimensional (2D) graphene and its lower-dimensional analogue, graphene nanoribbon (GNR) possible to be synthesized in different orientations. Few-layered graphene surfaces have also been used as electrodes for measuring electron transport through molecular films or single molecular nanojunctions $[29,30]$. The high conductivity and transparency of graphene 
electrodes have been utilized in organic FETs or organic PVCs [31-33].

Compared to 2D graphene, which is a zero-gap semiconductor, 1D nanoribbons (GNRs) are distinct by their electronic and optical properties. The electronic properties of GNRs are sensitive to many factors, edge modification being one of the main controls over them [25,34-37]. It has been shown that the zigzag-edged GNRs (zGNRs) are mainly low bandgap semiconductors in their ground state antiferromagnetic spin configurations [36]. However, they become half-metallic under the application of an external electric field [38,39]. The origin of magnetism in zGNRs stems from the localized edge states where the magnetic centres are ferromagnetically coupled. However, the two magnetic edge states couple antiferromagnetically resulting in poor magnetic moment in the ground state $[40,41]$. There have been numerous experimental and theoretical research studies on the charge and spin transport properties of zGNR heterojunctions [42-48] which show negative differential resistance (NDR), spin filtering effect, etc. These exciting properties make those materials highly promising to be used in different magnetoelectronic and spintronic devices $[11,49]$. On the other hand, armchairedged GNRs (aGNRs) exhibit semiconducting properties, where the band-gap decreases with increase in ribbon width $[36,37,50]$. This restricts the usability of aGNRs as conducting electrodes in molecular nanojunctions. However, defect formation or proper doping of heteroatoms like $\mathrm{N}$ or B shifts the Fermi level of aGNRs resulting in their metallic nature and thus, they have been properly used as the contact leads [34,51-56].

Charge transport properties of PCn-GR (graphene flakes) nanojunctions have recently been studied theoretically by employing density functional theory (DFT) in conjunction with the non-equilibrium Green's function formalism [11,19]. Pshenichnyuk et al [19] have demonstrated how the transport properties of these nanojunctions are mediated by PCn-GR coupling pattern and energy level sequences. Efforts have been made to tune the molecular energy levels of PCn by the influence of electron donating or withdrawing functional groups, which in turn affect the charge transport properties. Additionally, the particular effects of spin channels, which are crucial for zGNR leads, have also been resolved recently [11]. Thus, the current-voltage $(I-V)$ characteristics of differently functionalized PCn-GR/GNR nanojunctions show various types of spin resolved transport properties which are of prime interest for designing spintronic devices. Although the higher acenes are predicted to be less stable, recently, platelet-shaped crystals of hexacene have been prepared and an FET made of hexacene single crystal has shown a hole mobility much higher than that of PCn [18]. Other oligoacenes and even oligothiophenes are also reported to show high charge transport properties. The planar, rigid molecular backbones arising due to highly conjugated structures of those molecules are mainly responsible for their high conductivity [20]. Apart from acenes, a large number of heteroatom-doped acenes (heteroacenes) are also synthesized for their possible applications in organic electronics and photovoltaics. Recently, novel low band gap-fused thiophene-pyrrole-based heteroacenes (S,N-heteroacenes, $\mathrm{SN} n$ ( $n=$ number of fused aromatic rings)) have been successfully used as hole transporters in perovskite-based solar cells [20,57-60]. Owing to their high conducting nature, such materials have been shown to improve the photocurrent efficiency (PCE) of the solar cells to a great extent [58].

Noteworthily, such kind of organic semiconductor molecules can be categorized into two main groups. In the molecules, p-channels are introduced into the composite nanojunction, i.e., if holes are generated at the highest occupied molecular orbital (HOMO) levels, they are termed as hole transporters; on the other hand, if electrons are injected into the lowest occupied molecular orbital (LUMO) levels, thereby introducing n-type of effect, they are termed as electron transporters. Most of the stable known organic semiconductor materials are of p-type mostly because they are found to be stable only under ambient conditions [61]. The reported carrier mobilities of p-type materials are also higher in comparison with that of n-type materials $[5,62]$. The major cause behind the unavailability of high performing n-type organic semiconductor materials is the unavailability of suitable electron withdrawing functional groups which could stabilize the materials with the desired electronic energy levels [63]. Injecting an electron into the LUMO of an organic semiconductor is equivalent to the electrochemical reduction at the cathode. Furthermore, the injected electron at the LUMO (which is generally high energetic) is highly susceptible to react with aerial $\mathrm{O}_{2}$ and $\mathrm{H}_{2} \mathrm{O}$ under ambient conditions, finally leading to the annihilation of electron transport. Thus, the primary criterion for realizing a stable electron transporting material is the stabilization of its LUMO level to sufficient extent [63-66].

Although n-type organic semiconductors are rare, they are of complementary interest for the fabrication of $p-n$ junctions as well as other logic circuits [51,67]. Stable n-type organic semiconductors have recently been realized by introducing electron withdrawing groups into thiophene- or acene-based oligomers which are intrinsically hole-transporting materials [68-72]. Such kind of carrier switching may also occur through stereo-electronic changes inside a molecule [73,74]. However, in any case, the conformational change or incorporation of an electron-withdrawing group practically lowers the LUMO levels of the molecules, thereby provoking the crossing of the energy barrier for electron conduction [74-78]. Obtaining or predicting such electron conducting materials, even in silico, is still really demanding. Two methods have been singled out for converting a hole conducting material to an electron conducting; one is increasing the degree of polymerization and the other is chemical functionalization with a suitable electron withdrawing group as already discussed. Various experimental techniques, such as atomic force microscopy, scanning tunnelling microscopy (STM) in combination with the molecular break junction (BJ) method have been designed which can characterize the carrier type 
either through Hall measurement or through the detection of Seebeck coefficient. In the experimental set-up, the molecule is selectively placed in between a static metal surface and a movable STM tip [79-81]. Theoretically, one can intuitively predict the carrier type by proper and accurate molecular orbital (MO) analysis and following the transmission eigenchannels projected over the molecular states $[69,74,76]$.

Besides the spin and charge transport properties of acenes and heteroacenes, a large number of studies have also been devoted to show their photovoltaic activities [82-85]. Here also, PCn plays a leading role. Although solution processability of PCn is severely limited due to its poor solubility in water, design and synthesis of functionalized PCn are of recent interest with the aim of fabricating low cost solar cell devices. In a recent work, Pramanik et al [82] have synthesized a water-soluble PCn derivative with a low optical band-gap of $1.95 \mathrm{eV}$ which shows a moderate PCE, while fabricating a bilayer solar cell with $\mathrm{C}_{60}$ as an acceptor. Besides, differently functionalized $\mathrm{PCn}$ thin films are also reported to show intense optical absorption bands in the visible region, showing their promise in solar energy harvesting processes [86]. In this context, $\mathrm{PCn}-\mathrm{C}_{60}$ nanoadducts are important model systems to be studied theoretically as well as experimentally.

Among other different $\pi$-conjugated systems, oligothiophenes comprising donor-acceptor (D-A) or A-D-A types of architecture are well developed and are proved to show high performances in vacuum or solution-based organic solar cells [87-90]. As already discussed, the rigid and co-planar backbones of those systems easily suppress the conformation disorder which reduces the reorganization energy, finally enhancing their intrinsic charge mobility [91]. Similarly, $\mathrm{SN} n$ and their derivatives of similar nature have also been reported to show good photovoltaic performances [59]. Nalkylated S,N-PCn (SN5) molecule was synthesized a few years back as a component of fused thiophenes [92]. However, for their applicability in organic solar cells, different acceptor group functionalized SN5 derivatives have recently been studied. These derivatives in conjugation with different fullerene acceptors achieve remarkable PCE in vacuumprocessed or solution-processed solar cells [20,57]. Even, some of these materials show up to $10.3 \%$ of PCE when applied as a hole transporter in perovskite-based solar cells [58]. Dicyanovinyl (DCV)-substituted SN5 (DCV-SN5) has been shown to produce PCE up to $5.64 \%$ which is higher than that of SN6 analogue $(\mathrm{PCE}=3.02 \%)$ with $\mathrm{C}_{70}$ as the counter composite [93]. A higher PCE has also been achieved with further modifications at the edge terminations of the molecules or by applying thermal annealing and subsequent solvent vapour annealing techniques [93-96]. As for example, Wetzel et al [96] have developed a DCV-SN6-based vacuum-processed planar-heterojunction with an excellent PCE of $7.1 \%$ with $\mathrm{C}_{60}$ as an acceptor. In the meantime, the same group has extended the synthetic protocol up to SN10 [60]. As extended delocalization in $\mathrm{SN} n$ shows better visible region optical absorption properties, it is expected that such systems may produce even better PCE. Very recently, on the basis of systematic $a b$ initio simulations, our group has studied the optoelectronic properties of such materials in view of their potential applications in small moleculebased solar cells [85]. It has been demonstrated that there has been an even-odd parity in the intramolecular charge separation process, which is again reflected in generating charge transfer (CT) states while making composite systems. As a matter of fact, the odd $\mathrm{SN} n$ molecules show faster CT rates, thereby showing better photovoltaic performances [85].

Suitable electron acceptors also play an essential role in the performance of solar cell devices. Although semiconductor metal oxide surfaces like $\mathrm{TiO}_{2}$ and $\mathrm{ZnO}$ are well known materials in this regard [97-109], $\mathrm{C}_{60}$-fullerene and its derivatives are now being utilized as excellent electron acceptors because of their charge capturing ability originating from their unique structural rigidity and high degree of symmetry [110-113]. The suitable reduction potentials and the small reorganization energy during the electron-transfer processes are also very important for their successful implementations in bulk-heterojunction (BHJ) solar cells $[114,115]$. Fullerene derivative, particularly, phenyl- $\mathrm{C}_{61}$-butyric acid methyl ester $\left(\mathrm{PC}_{61} \mathrm{BM}\right)$ is the mostly used acceptor counterpart in this regard [116]. It is known that the injection of photoexcited electron from $\mathrm{D}$ to the A counterpart is the most crucial step in an organic photovoltaic device. To facilitate such process, it is required that the LUMO level of D must lie above the LUMO or conduction band of A. On the other hand, the HOMO level of $\mathrm{D}$ should be at lower energy position with respect to the chemical potential of the counter conductive electrode so as to facilitate the regeneration of the oxidized dye [117,118]. As a whole, a type-II band energy alignment is desired to have a good photovoltaic performance [111,112]. The energy alignment of $\mathrm{PC}_{61} \mathrm{BM}$, in most of the cases, provides such favoured situation, thereby enhancing the power conversion efficiency of the devices [113]. It has been demonstrated that in many cases, acene- $\mathrm{C}_{60} \mathrm{D}-\mathrm{A}$ type of composites show ultrafast CT dynamics and slow recombination dynamics [85,119-125].

The above literature briefly outlines the prospects of acenes and heteroacenes in different optoelectronic devices which involve charge transport or CT through such molecular nanojunctions or nanocomposites. The present review aims to point out some interesting prospects of acenes and heteroacenes for favouring the CT and charge transport phenomena. Electron transport being a junction property, we will outline some important aspects of molecular nanojunctions comprising individual acene/heteroacene molecules and graphene or gold surfaces. The photovoltaic properties of the molecules would be discussed on the basis of molecular heterojunctions with fullerenes and their derivatives. The CT dynamics of such composite systems would be discussed on the basis of Marcus theory of electron transfer [126,127] and also with the help of time-dependent DFT (TDDFT) analysis. 



Figure 1. Optimized ground-state geometries of the single $\mathrm{PCn}$ molecule (left) and PCn-DA- $\mathrm{C}_{60}$ composite system (right) [84]. The bond lengths are presented in $\AA$.

\section{Geometry and electronic structure}

\subsection{Pentacene and its derivatives}

In general, the $\pi$-conjugated aromatic molecules are planar in nature up to a certain extent of ring fusion. However, the planarity in the molecular backbone decreases with increase in molecular length. Likewise, PCn is planar in its ground state with a $D_{2 h}$ symmetry. The generalized gradient approximation (GGA)/PBE calculations report that in-plane perpendicular $\mathrm{C}-\mathrm{C}$ bond distances of $\mathrm{PCn}$ vary from 1.43 to $1.46 \AA$, while entering from edge to the centre of the molecule (figure 1) [84]. The zigzag $\mathrm{C}-\mathrm{C}$ bond lengths also vary within the range from 1.38 to $1.44 \AA$, however, the sum of two such bonds in a hexagon is maintained at 2.82 $\AA[11,128,129]$. Now, in the case of substituted PCn, the substituent groups $\left(\mathrm{NO}_{2}, \mathrm{NH}_{2}\right)$ are essentially in the same molecular plane thereby facilitating the extended delocalization. In that case, the symmetry as well as the $\mathrm{C}-\mathrm{C}$ bond distances are changed to some extent. For practical implementation in solar cells, the solubility of PCn needs to be increased [82] and in view of this, carboxylic acid derivatives are prepared. The carboxylic acid groups also play the role of anchoring group for making composite system with $\mathrm{C}_{60}$. It has been reported that the water soluble PCn diacids ( $\mathrm{PCn}$ DA) and their salts are relatively robust in the context of solar cell applications. They possess a higher degree of thermal stability as well as superior resistivity towards aerial oxidation [82].

Back to back experimental and theoretical studies have been performed on the geometry and optoelectronic properties of a series of substituted PCn derivatives. All of the derivatives, 1-12 (figure 2) were synthesized, purified and then characterized by various spectroscopic and cyclic voltammetric (CV) methods [130]. The theoretically calculated HOMO-LUMO gap of PCn is reported to be $1.1 \mathrm{eV}$ $[11,128]$ which is somewhat less than the experimental value $(1.82 \mathrm{eV})$ reported from ellipsometric spectra of thin PCn films [131]. Such a discrepancy between the theoretical and experimental band gap values may be attributed to the use of GGA in exchange correlation functional [11,84]. However, for the other substituted PCn derivatives, the calculated HOMO and LUMO energies and the corresponding gaps complement the experimental data [130] as could be found from table 1. The gas phase calculations on PCn-DA by using B3LYP/6-31g(d) or B3LYP/6-311g(d,p) levels of theory indicate a ground state geometry with $C_{1}$ symmetry [84] which corroborate well with the experimental observation as reported from X-ray crystallographic data [82]. The calculated HOMO-LUMO gap of PCn-DA is in the order of $2.1 \mathrm{eV}$ using different levels of theories [84] which also matches well with the available experimental data [82]. Noteworthily, the relative positions of the HOMO $(-5.16 \mathrm{eV})$ and LUMO $(-3.08 \mathrm{eV})$ are very significant for their optoelectronic uses. However, the positions of these frontier orbitals are tunable by introducing different electron withdrawing or donating groups. Thus, one may design novel composite materials for photovoltaic purposes $[82,84,130]$.

\section{$2.2 S, N$-heteroacenes}

As already discussed, a series of $\mathrm{SN} n$ molecules ranging from $n=3-10$ has been synthesized and characterized by using NMR spectroscopy and mass spectrometric techniques [60]. The representative model structures of such species are found in figure 3 . On the basis of the structural feature, the molecules can be categorized into two main subgroups: even heteroacenes, $\mathrm{SN}(2 m)(m=2-5)$ containing thienothiophene units and having a $C_{2 h}$ symmetry, and odd heteroacenes, $\mathrm{SN}(2 m+1)(m=1-4)$ containing alternating thiophene and pyrrole rings all of which possess a $C_{2 \mathrm{~V}}$ symmetry. SN7' is the analogous of SN7, but it differs from the latter by the number and sequence of the thiophene and pyrrole rings. All the $\mathrm{SN} n$ molecules exhibit a rigid planar structure which indicates a thorough delocalization along the molecular backbone. However, a slight bond-length alteration (BLA) is observed at the inner parts of the molecules $[59,85]$. For the lower $\mathrm{SN} n$, the observed BLA is on the order of $0.014 \AA$ which is slightly lower than that of the corresponding oligothiophenes [69]. However, with increase in conjugation length, the extent of BLA decreases. Functionalizing the molecules with electron withdrawing groups (such as DCV) at the both ends, it increases the extent of delocalization which further reduces the BLA. For example, for DCV-SN9 and DCV-SN10, the BLA is too small (on the order of $0.005 \AA$ only) indicating 'nearly complete equalization' in the inner part of the conjugated backbone [60].

The computed HOMO, LUMO energy levels and the associated gaps of the substituted and unsubstituted $\mathrm{SN} n$ 


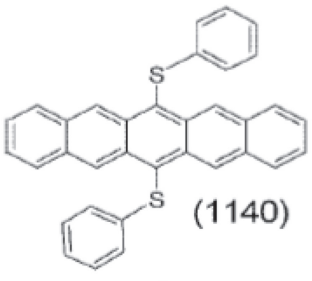

1

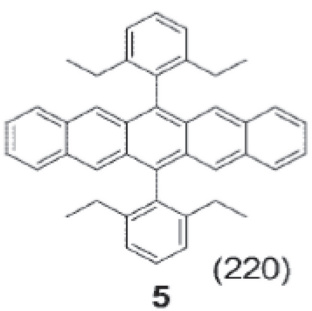

5

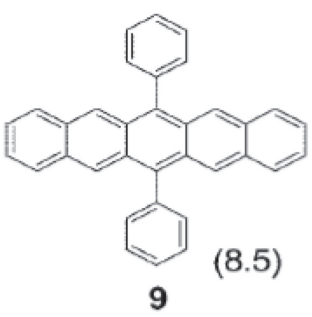

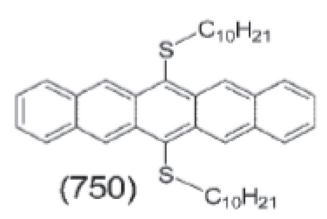

2

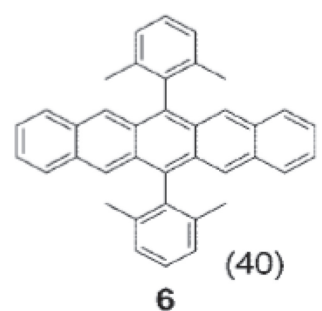

6

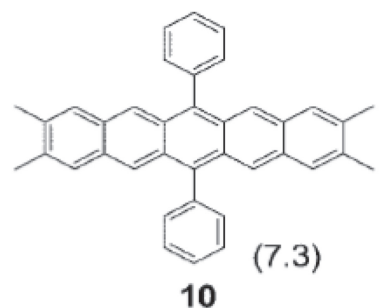<smiles>Cc1cccc(C)c1-c1c2cc3cc(Cl)c(Cl)cc3cc2c(-c2c(C)cccc2C)c2cc3cc(Cl)c(Cl)cc3cc12</smiles>

3



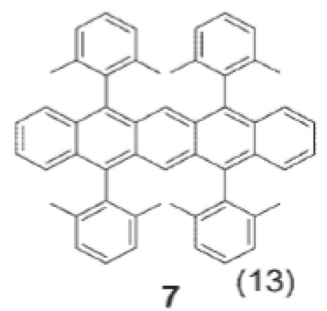<smiles></smiles><smiles>COc1cc2cc3c(-c4ccccc4)c4cc5cc(OC)c(OC)cc5cc4c(-c4ccccc4)c3cc2cc1OC</smiles>

11



12

Figure 2. Structures of some PCn derivatives (1-12) with their associated half-lives in minutes (shown in parentheses) [130].

molecules using the HSE06/6-31G** level of theory are displayed in figure $4 \mathrm{a}$. The corresponding experimental results obtained from the $\mathrm{CV}$ measurement are also shown in figure $4 \mathrm{~b}$ for the comparison. Note that for experimental case, the pyrrole rings are functionalized by the hexyl group which, however, affect the energy gap marginally $[60,85]$. It is interesting to observe that the computed HOMO-LUMO gaps [85] for the unsubstituted $\mathrm{SN} n$ match excellently with the $(\mathrm{CV})$ results [60], although, the individual positions of the HOMO and LUMO differ slightly. The figure clearly indicates that the HOMO-LUMO gap decreases with increase in the conjugation lengths of $\mathrm{SN} n$. A similar trend is also observed for the substituted $\mathrm{SN} n$ molecules with increasing strengths of electron withdrawing groups $[59,93]$. This is probably driven by the prominent $\mathrm{CT}$ interactions between the rigid molecular backbone of the $\mathrm{SN} n$ and the attaching electron withdrawing groups at the two ends [59]. The tunability of such electronic gap as a function of molecular length and the nature of substituent group can be utilized properly for solar energy harvesting processes [85].

\section{Transport properties of the molecular nanojunctions}

We already introduced that the transport properties of molecular nanojunctions depend on the molecule-lead coupling pattern. Although the transmission channels are determined from the individual molecular states, their relative positions are guided by the nature of the electrodes that are used. Here, we will show the effect of both types of electrodes, graphene and metal surfaces, mainly Au(111) surfaces. For PCn molecular junctions, people mainly think of PCn-graphene nanojunctions but for the $\mathrm{SN} n$ molecules, molecule-Au(111) nanojunctions are considered. The reason is so simple because the PCn molecule can be connected with a variety of modes with graphene, whereas the $\mathrm{SN} n$ molecules are more susceptible to make molecular contacts with metal surfaces $[11,19,69,76,132]$.

\subsection{Pentacene-graphene nanojunction}

Before going to the details of the transport properties of PCn-graphene (mainly GR or GNR) nanojunction, let us recapitulate that the ground state of $\mathrm{zGNR}$ is antiferromagnetic with a zero net magnetization and the spin degenerate edge states are separated by a small energy gap around the Fermi level [37,38]. The GGA/PBE calculated band-gap for 4-zGNR is on the order of $0.8 \mathrm{eV}$ [11]. Thus, although $\mathrm{PCn}$ is non-magnetic in nature, the overall system consisting of graphene fragments within the scattering region (SR) is treated with antiferromagnetic spin configurations. A model system for computing transport characteristics of such a nanojunction is shown in the top panel of figure 5 . 


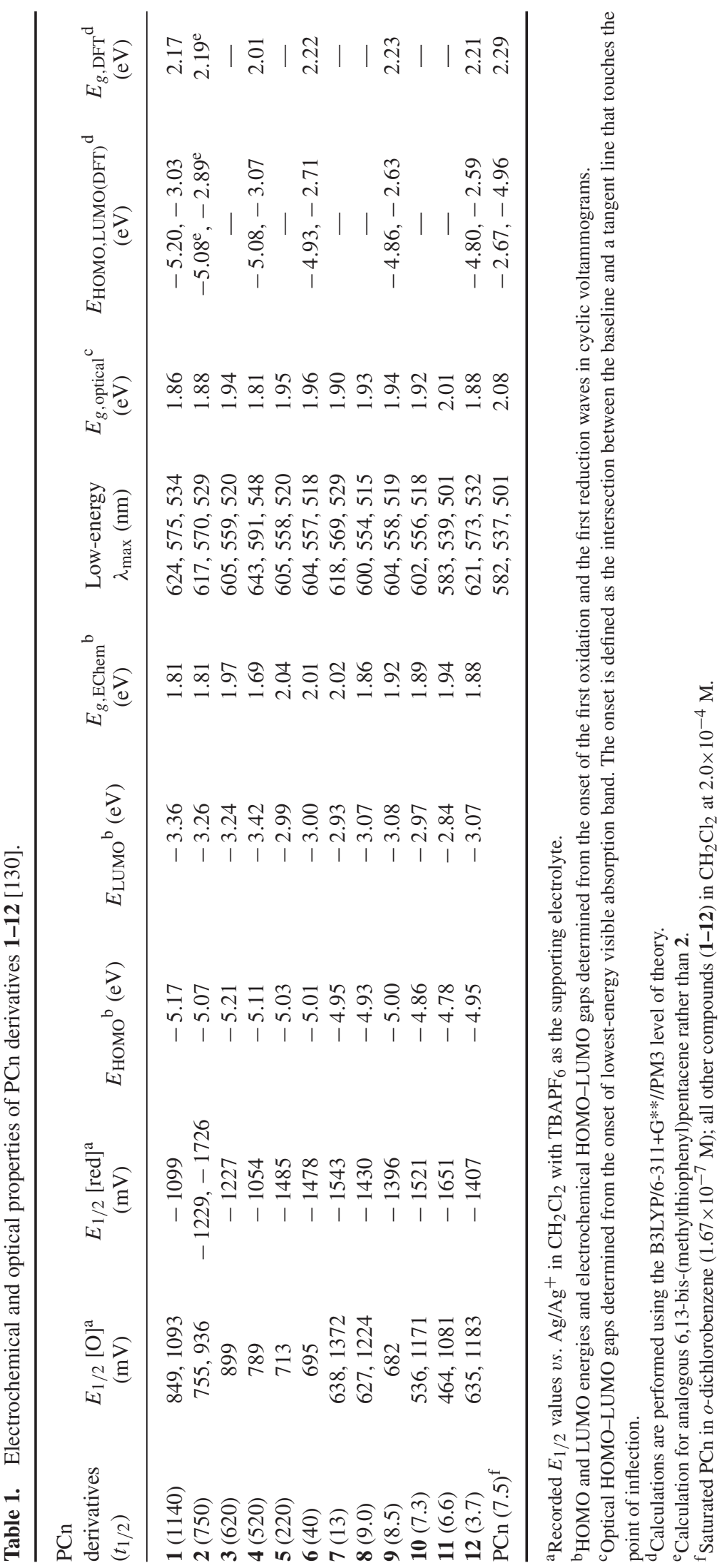




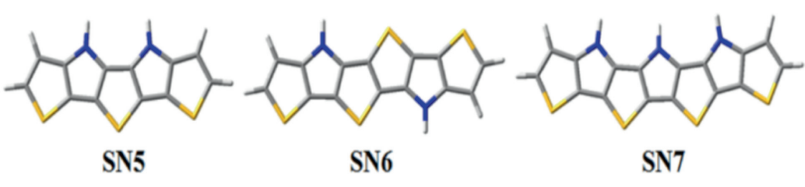



SN7'
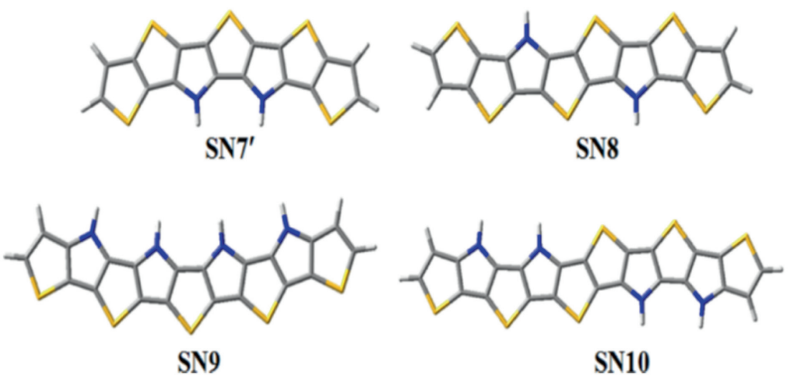

Figure 3. Optimized geometries of the $\mathrm{SN} n(n=5-10)$ molecules showing the sequence of fusion of thiophene and pyrrole rings [85].


Figure 4. HOMO-LUMO energies and their corresponding gaps for $\mathrm{SN} n$ and $\mathrm{DCV}-\mathrm{SN} n$ molecules of different conjugation lengths obtained from (a) computations using the HSE06/6-31G** level of theory [85] and (b) CV measurements [60].

It is basically a two probe model where the two edges of PCn or substituted PCn are connected with two semiinfinite electrodes (LHE/RHE, i.e., 4-zGNR in this case) via $\mathrm{H}$-terminated graphene fragments. However, in some
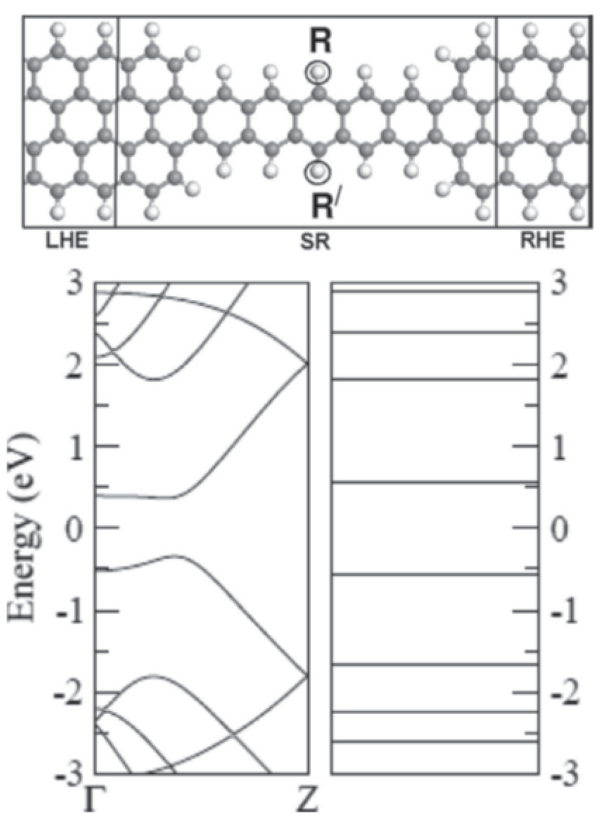

Figure 5. Optimized supercell structure (LHE-SR-RHE) of the PCn-zGNR nanojunction (top panel), band structure of 4-zGNR (bottom left panel), and energy levels of PCn molecule (bottom right panel). Grey and white coloured balls represent $\mathrm{C}$ and $\mathrm{H}$ atoms, respectively. The encircled $\mathrm{H}$ atoms in $\mathrm{PCn}$ indicate the places of substitutions. For energetic representation, the Fermi energy of individual system has been set to zero [11].

cases, the molecules are connected through amide linkages or via oxygen atoms [19]. The region in between the two electrodes, as a whole is known as SR. The coherent electron transport as a result of voltage difference between two electrodes is measured in terms of transmittance by using Landauer-Buttiker formula which utilizes nonequilibrium Green's function method $[55,133,134]$. The total integrated current can be expressed as

$$
I\left(V_{\mathrm{b}}\right)=\frac{e}{h} \int_{-\infty}^{\infty} T\left(E, V_{\mathrm{b}}\right)\left[f_{\mathrm{L}}\left(E, \mu_{\mathrm{L}}\right)-f_{\mathrm{R}}\left(E, \mu_{\mathrm{R}}\right)\right] \mathrm{d} E,
$$

where $f_{L(R)}$ is the equilibrium Fermi-Dirac distribution function for left (right) electrode, $\mu_{\mathrm{L}(\mathrm{R})}$ is the electrochemical potential of the left (right) electrode such that energy bias $e V_{\mathrm{b}}=\mu_{\mathrm{L}}-\mu_{\mathrm{R}}$, and $T\left(E, V_{\mathrm{b}}\right)$ is the transmittance of an incident electron with energy $E$ which can be obtained using the below equation:

$$
T\left(E, V_{\mathrm{b}}\right)=\operatorname{Tr}\left[\Gamma_{\mathrm{L}} G^{\mathrm{R}} \Gamma_{\mathrm{R}} G^{\mathrm{A}}\right],
$$

where $G^{\mathrm{R}(\mathrm{A})}$ is the retarded (advanced) Green's function of the $\mathrm{SR}$, and $\Gamma_{\mathrm{L}(\mathrm{R})}$ is the coupling strength of the left (right) electrode. 


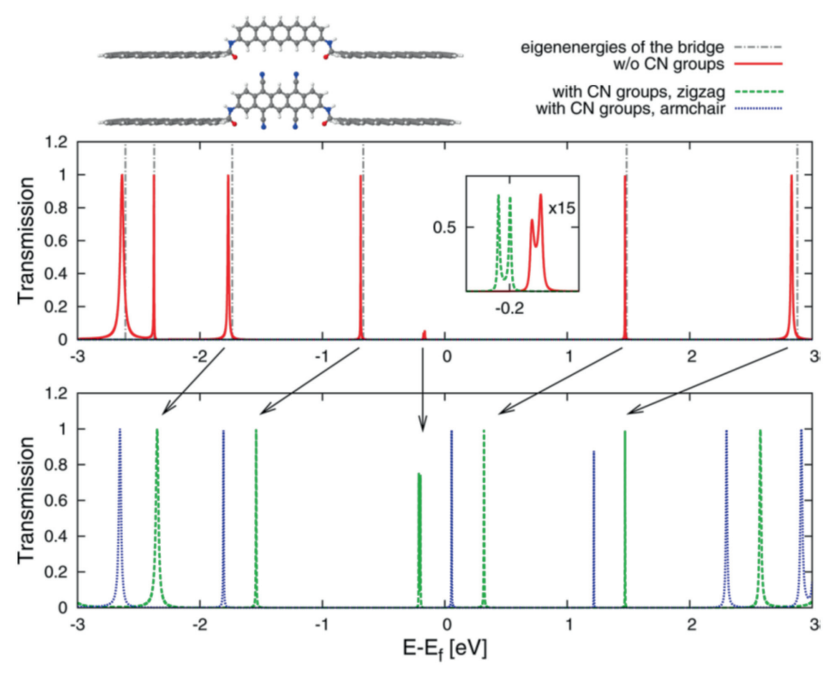

Figure 6. Transmission plots for weakly coupled PCn-GR nanojunctions (shown in ball and stick model); the top panel shows the unsubstituted PCn and the bottom panel demonstrates the effect of $\mathrm{CN}$ functionalization to that. The dashed-dotted lines in the top panel represent the corresponding eigenenergies. The differences between the zigzag and armchair nanocontacts are also shown by different colours. The inset highlights the edge-induced peak with and without CN substitution [19].

Figure 6 (top panel) shows the spin independent transmission function of the amide-linked PCn-GR nanojunction [19]. It exhibits a series of transmission peaks which can be classified into two groups. The high intensity (with a maximum value of unity) isolated transmission peaks which are referred as the molecular resonance transmissions and the low intensity double peak transmission which appears close to the Fermi energy. Each of the molecular resonance transmissions is always associated with some discrete energy level of the molecular bridge and hence appears close to that particular state. The widths of these peaks depend on the moleculeled coupling. On the other hand, the low-intensity peak close to the Fermi energy cannot be related directly to molecular eigenstates and this does not correspond to direct tunnelling between the GR electrodes, rather this is assigned as an additional transport channel induced by the edge states of the GR leads or simply edge-induced transport channel. Noticeably, similar edge-induced transport channels have also been reported in different molecular nanojunctions made of CNTs and other metal surfaces $[69,74,135-137]$. Low-bias conductance of the molecular junction is mainly guided by those transport channels due to their close proximity to the Fermi energy.

The intensity of the edge-induced transmission channels may however be enhanced if the molecular levels appear closer to the Fermi energy. For example, if the $\mathrm{PCn}$ is functionalized with $\mathrm{CN}$, it causes an overall shifting of the molecular eigenstates to a relatively lower energy levels. Consequently, the LUMO of PCn comes closer to the Fermi level

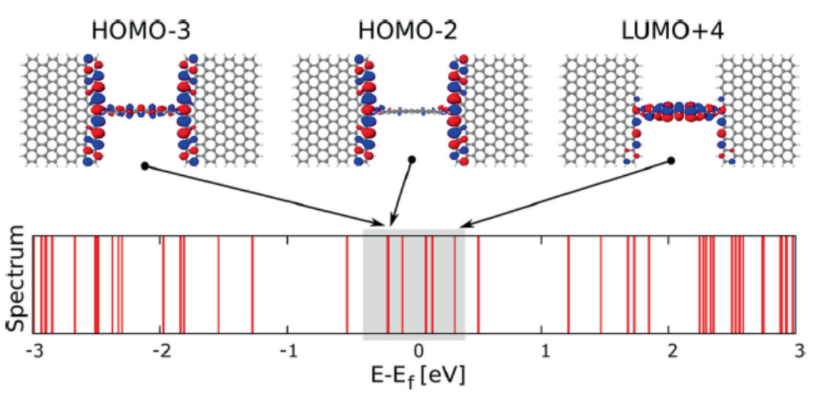

Figure 7. Energy spectrum of $\mathrm{CN}$-functionalized $\mathrm{PCn}-\mathrm{GR}$ nanojunction formed by the weakly coupled scheme through amidebinding groups. The highlighted area demonstrates the appearance of eight edge states (including HOMO-3 and HOMO-4) and one molecular state (LUMO+4, which has been embedded into the family of edge states through $\mathrm{CN}$-functionalization to the $\mathrm{PCn}$ ). The energies of the states indicated by arrow correspond to the peaks at $E=-0.2$ and $+0.2 \mathrm{eV}$, respectively [19].

of the electrode, thereby causing a better overlap between the molecular states and the edge-states of GR. This results in an additional intensity of the edge-induced transmission peaks as could be found in the bottom panel of figure 6 [19]. Figure 7 further analyses the nature of edge-induced transmission channels for the $\mathrm{CN}$-functionalized PCn-GR nanojunction. It shows the isosurface plots of the MOs of the overall nanojunction close to the Fermi energy. As found, these states can be classified into two groups: first, the states localized preferentially in the GR contacts (HOMO- 3 and HOMO-2) are nothing but the well-known edge states of zGNR $[38,138,139]$, and second, the molecular states mostly localized on the bridge (LUMO+4). It has been demonstrated that the edge-states, due to their localization at the moleculeGR interface, strongly interact with the molecular states and thus, induce the additional transport channels. For example, the transmission spectrum shown in figure 7 (bottom panel) demonstrates that for the $\mathrm{CN}$-functionalized $\mathrm{PCn}$, the edgeinduced transmission peak at $E=-0.2 \mathrm{eV}$ corresponds to the near-degenerate pair of states, HOMO-2 and HOMO-3. On the other hand, the transmission peak at $E=+0.2 \mathrm{eV}$ corresponds to the regular transmission channel caused by the state LUMO+4. To unambiguously prove that the appearance of edge-induced transmission channel is due to the edge states of zGNR nanocontacts, one can reproduce similar transport characteristics with aGNR nanojunctions. As aGNRs do not possess edge states [37,51,53], the transmission functions for such nanojunctions should differ. More precisely, the edgeinduced transmission channels are not expected to appear for those systems [19].

As already introduced, another important aspect of PCnGR nanojunctions is their spin-induced transport characteristics. Because zGNRs possess spin-degenerate edge states, external or internal influence of charge or magnetic dipole will surely lift that degeneracy and that would be reflected in the transport characteristics of the nanojunction. The 



Figure 8. Spin resolved $I-V$ characteristics of (a) pristine PCn-zGNR nanojunction, (b) nitro-amine-bifunctionalized PCn-zGNR nanojunction. Black and red lines represent the spin currents for spin-up and spin-down channels, respectively. For the pristine one, the two spin currents are inseparable. (c) Spin resolved transmission spectra of nitroamine-bifunctionalized PCn-zGNR nanojunction. Vertical dotted red lines indicate bias window [11].

computed spin-induced transmission characteristics of such nanojunctions consisting pristine and dipolar group-functionalized PCn are shown in figure 8. The small transport gap of zGNR close to the Fermi level, in each case, is attributed to the band opening of the zGNR leads [11]. For pristine $\mathrm{PCn}$ molecule, the spin channels are almost indistinguishable, however, in the case of the substituted PCn, two spin components are resolved as found in figure 8c.

For the unsubstituted $\mathrm{PCn}$, the observed $I-V$ curve (figure $8 \mathrm{a}$ ) is antisymmetric in nature since the overall SR has a mirror symmetry. As already discussed, the low-bias conductance is mediated by edge-induced transmission channels producing a very low current $(I)$. After the threshold voltage (half of the band-gap energy), the current increases by the influence of HOMO or LUMO resonating channels. With increasing the applied bias, the current increases up to $2.2 \mathrm{~V}$ and thereafter, it falls within the bias ranging from 2.2 to $2.6 \mathrm{~V}$. This indicates a NDR behaviour of the PCn-GNR nanojunction as also commonly observed for many GNR contacts [51,139-141]. The observed NDR phenomenon is attributed to the localized states of PCn molecule (figure 5) [11,142]. For a large applied bias, there occurs asymmetric coupling between the molecular states and the electrodes and this causes the appearance of asymmetric potential near the source electrode. As a consequence, there occurs strong localization of molecular states along the different parts of the SR which ultimately reduces the transmission coefficient and hence, the current [142]. Such kind of NDR phenomena may also originate from the poor coupling between the doped leads and the discrete molecular states where the doped leads produce narrow density of states (DOS) [140].

Figure $8 \mathrm{~b}$ displays the $I-V$ characteristics of PCn-zGNR nanojunction where $\mathrm{PCn}$ is functionalized by both nitro and amine groups at $\mathrm{R}$ and $\mathrm{R}^{\prime}$, positions, respectively, as shown in figure 5. Figure $8 \mathrm{c}$ depicts the transmission spectra of the corresponding nanojunction at different applied biases. At some finite bias $(0.8 \mathrm{~V})$, the stronger transmission peak at the Fermi level is expected due to the contribution from the edge states. However, with increasing the bias, this peak disappears, instead, two peaks of similar intensity appear. Interestingly, the up and down spin components are resolved beyond the threshold voltage, resulting in different spin currents. However, both the spin currents show NDR behaviour, but with different peak positions and different peak-to-valley ratios [11].

The origin of the spin resolved transport characteristics of the PCn-zGNR nanojunction may be understood on the basis that under transverse electric field, zGNRs exhibit half metallic property $[36,143]$ which stems from a unique interplay of the edge magnetizations, their antiferromagnetic coupling and 
as a consequence, relative potential shifting between the two edges of the zGNRs. Apart from external electric fields, this may also happen due to other causes such as, introduction of quantum dots at the zigzag edges, substitution of the edge carbons by boron, nitrogen, etc., functionalization of the zGNR edges or by deposition of ferroelectric materials on the zGNR surface [38,144-146]. So, it is unambiguous that proper functionalization of PCn with dipolar groups will affect the edge magnetizations of zGNR. As a consequence, the edge induced spin states are resolved upon the application of external bias voltage. This argument can easily be proved by proper choice of functional groups and also by varying the positions of functionalization. When PCn is functionalized with a relatively weak electron withdrawing or donating group, which obviously have low dipolar effect, the spin resolution is found to be weak [11]. Even, when the two same groups are attached at the opposite sites of $\mathrm{PCn}$ (substituted at $\mathrm{R}$ and $\mathrm{R}^{\prime}$ positions as shown in figure 5), the dipolar effect is nullified and thus, unpolarized transport properties are observed [11,19]. In contrast, a more prominent spin resolved transport properties are observed when the PCn molecule is functionalized simultaneously with nitro and amine groups at two opposite sides [11]. In that case, a pronounced dipolar effect as a result of opposing electronic effects of the nitro and amine groups produces severe spin resolution in the transmission channels $[11,145]$. The consequence of spin resolution in the transport properties is the spin filtering ability by the properly functionalized PCn-GR nanojunctions, which can be quantified in terms of spin resolved current at finite bias $\left(I_{\mathrm{s}}\right)$ and is defined as $I_{\mathrm{s}}$ $=\left(I_{\mathrm{dn}}-I_{\mathrm{up}}\right) /\left(I_{\mathrm{dn}}+I_{\text {up }}\right)$. The highest value of $I_{\mathrm{s}}=0.41$ is reported at an applied bias $V_{\mathrm{b}}=2.6 \mathrm{~V}$ for nitro-amine bifunctionalized PCn [11].

\subsection{S,N-heteroacene-Au(111) nanojunction}

$\mathrm{SN} n$ molecules could be anchored to Au-surface through various modes. The transport properties of molecular nanojunction strongly depend on the anchoring groups through which the molecules are connected with the electrodes [147]. For anchoring a molecule with metal surfaces, in general, thiols/thio-ethers, amines, carboxylic acids, sulphides, phosphides, etc. are used. The overall transport properties of a molecular nanojunction thus must be influenced by the electronic coupling between the molecule and the electrodes. The stronger the coupling between the discrete energy levels of the molecule and the continuum of metal sub-bands, the larger the broadening of transmission resonances close to the Fermi surface and consequently, the nanojunction would be more conducting. The use of thiols or thio-ethers as anchoring groups is advantageous because of their flexibility as required in STM-BJ experiments. In these instances, the optimized $\mathrm{Au}-\mathrm{S}$ bond lengths are reported in the range of 2.29$2.39 \AA$ [148-151]. However, direct Au-C covalent linkages are also reported in the literature showing highly conducting molecular nanojunctions [152-154]. On the other hand, $\mathrm{Au}-\mathrm{N}$ contacts have recently been reported for the amine



Figure 9. Optimized geometries of the metal-molecule contacts for the pristine (a) $\mathrm{SN} n$, (b) DCV-SN $n$ and (c) $\mathrm{SMe}-\mathrm{SN} n$ ( $\mathrm{SN} 5$ as a representative) molecules [157].

or cyano-terminated molecules with an appreciable amount of resonance transmission [151]. In case of amines, an $\mathrm{Au} \longleftarrow \mathrm{N}$ dative bond (average bond distance $2.35 \AA$ ) is formed by the donation of lone pair of electrons over the $\mathrm{N}$ atom to the vacant d-orbital of a suitable $\mathrm{Au}$ atom of the $\mathrm{Au}(111)$ surface [155], while for the latter case, an $\mathrm{Au}-\mathrm{N}$ covalent bond is formed with an average bond distance of $2.15 \AA[151,156]$. Some model nanojunctions (Au-molecule$\mathrm{Au}$ ) consisting of pristine and substituted $\mathrm{SN} n$ with $\mathrm{Au}(111)$ surfaces are shown in figure 9 [157].

The Au-molecule-Au nanojunctions constituted of pristine $\mathrm{SN} n$ and their different derivatives, especially DCV-SN $n$ show quite interesting transport phenomena. The DCV-SN $n$ molecules, where two DCV groups are attached at the two ends of the $\mathrm{SN} n$ molecules, have recently been synthesized with a narrower band gap and high thermal stability $[59,60,85]$. DCV being strong electron withdrawing in nature, imparts a lower HOMO-LUMO gap and as a consequence, a red-shifting is occurred in the intramolecular CT band which in turn helps to improve the photocurrent of the devices [95]. Besides, other derivatives of SN5 also show promise as prominent hole transporters and absorbers in perovskite-based solar cells with moderate and high PCE [58,158,159]. Another member of this series, DCV-SN6 has also very intense optical transition. DCV-SN6 is reported to exhibit good p-channel charge-carrier mobility, up to $0.02 \mathrm{~cm}^{2} \mathrm{~V}^{-1} \mathrm{~s}^{-1}$ as a consequence of an extended band-like $\pi$-conjugated backbone [59]. Noteworthily, while comparing the conducting properties of the $\mathrm{SN} n$ molecules as a function of conjugation length, it has to be kept in mind that the $\mathrm{SN} n$ molecules show a difference in their heteroatomic orientation and ordering for the even and odd number of fused rings (figure 3). This orientation difference leads to a different extent of 


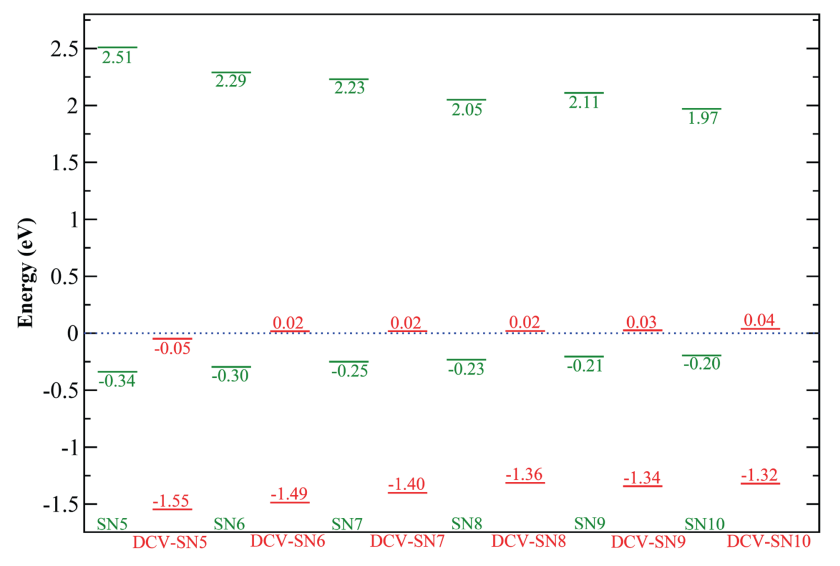

Figure 10. Low-lying MPSH eigenvalues of the $\mathrm{SN} n$ and DCV$\mathrm{SN} n$ molecular junctions at zero applied bias [157].

intramolecular charge separation [85]. However, in both the cases, the molecular conductance $(G)$ decreases exponentially with increase in conjugation length $(l)$. Obviously, one gets a linear plot of $\ln G v s . l$ and the negative slope of the straight line gives the conductance decay constant $(\beta)[74,132,160]$. For the odd $\mathrm{SN} n$ molecules, $\beta=0.06 \AA^{-1}$, while for the even ones, it is $0.04 \AA^{-1}$. The $\beta$ values for the odd and even DCV-SN $n$ molecules are reported to be 0.29 and $0.10 \AA^{-1}$, respectively, and those for the odd and even $\mathrm{SMe}-\mathrm{SN} n$ molecules are 0.23 and $0.12 \AA^{-1}$, respectively [157]. In each case, the odd ones show relatively higher $\beta$ values in comparison with that of the even ones. However, the computed $\beta$ values for the $\mathrm{SN} n$ and $\mathrm{DCV}-\mathrm{SN} n$ molecules are to some extent lower in comparison with that of the oligothiophene or oxidized oligothiophene chains $[74,132]$.

Now, the conductance characteristics of the molecular junctions could be known from the relative positions of the highest occupied and lowest unoccupied Kohn-Sham states projected over the molecules with respect to the Fermi energy of the metal electrode. These are nothing but the eigenstates of the molecular-projected self-consistent Hamiltonian (MPSH states). Figure 10 shows the zero bias eigenvalues of those frontier eigenstates projected over the $\mathrm{SN} n$ and DCV-SN $n$ molecules with respect to the Fermi energy of $\mathrm{Au}(111)$ surfaces as electrodes. These are the possible eigenchannels through which electron transport may occur. As could be found from figure 10, for the SN5-Au molecular contact, the projected HOMO and LUMO energy positions are -0.34 and $2.51 \mathrm{eV}$, respectively. The corresponding values for DCV$\mathrm{SN} 5$ are -1.55 and $-0.05 \mathrm{eV}$, respectively. Clearly, HOMO of SN5 and LUMO of DCV-SN5 are closer to electrode Fermi energy and thus, they serve as the main transporting channels for the corresponding molecular nanojunctions. Similar situations are arrived for the other pairs of $\mathrm{SN} n$ and DCV-SN $n$ molecular junctions with similar molecular lengths. Now, for $\mathrm{SMe}-\mathrm{SN} n$ molecular junctions, the relative energy positions of the eigenstates are changed but still, the HOMO levels are closer to the Fermi level, at least for the longer chains.

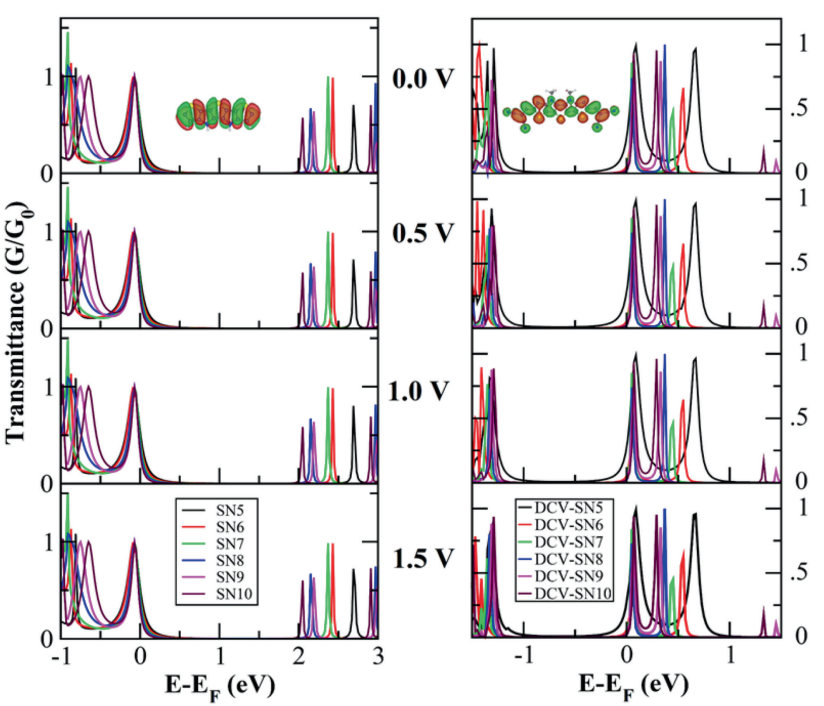

Figure 11. Zero and finite-bias transmittance of the $\mathrm{SN} n$ (left panel) and DCV-SNn (right panel) molecular junctions as a function of relative Fermi energy. One of each isosurface plots of the MPSH eigenchannels of the $\mathrm{SN} n$ and DCV-SN $n$ molecular junctions at zero applied bias are shown to indicate their distinct characteristics as molecular HOMO and LUMO levels, respectively [157].

Figure 11 shows the transmittance plots of the unsubstituted and DCV-substituted $\mathrm{SN} n$ molecular junctions (left and right panels, respectively). It is clear from the figure that at a particular applied bias, the major transmission peaks at both sides of the Fermi level (set to zero energy) correspond to the molecular HOMO and LUMO levels. The figure clearly indicates that the closest transmission peaks to the Fermi energy, in the case of $\mathrm{SN} n$ nanojunctions, correspond to the HOMO resonating channels, while those for the DCV-SN $n$ nanojunctions, correspond to the LUMO resonating channels. For the $\mathrm{SMe}-\mathrm{SN} n$ nanojunctions, there is a transmission gap around the Fermi level, however, some low intensity peaks are appeared in between the two major peaks. These are referred to the metal-induced transmission channels $[11,74,75]$ which are mainly responsible for their low-bias transport properties. To demonstrate the fact, one can obtain the isosurface plots of the transmission eigenchannels (MPSH states) at different applied biases. It has been revealed that for the pristine $\mathrm{SN} n$ molecules, the lowest transmission eigenchannels in the low bias region correspond to the molecular HOMO states. Although there are slight differences between the MPSH eigenchannels and the corresponding HOMOs in the case of $\mathrm{SMe}-\mathrm{SN} n$ nanojunctions, they mostly resemble HOMO, not the LUMO nor even the HOMO-1. So, the $\mathrm{SN} n$ and $\mathrm{SMe}-\mathrm{SN} n$ molecules are mainly hole transporting materials. In contrast, the lowest MPSH states corresponding to the DCV-SN $n$ molecules corroborate with the LUMO levels of the respective free molecules, clearly indicating the n-type of conductance. What happens is that, the strong electron withdrawing DCV group pulls down the LUMO energies 
of the DCV-SNn molecules as compared to those of the corresponding unsubstituted ones. Thus, the LUMOs become closer to the Fermi-level of the electrode and this facilitates electron transport from the metal electrode to the vacant orbitals of the molecules. These clearly reveal that the $\mathrm{SN} n$ or $\mathrm{SMe}-\mathrm{SN} n$ molecules are hole conducting, while the DCV terminations at both ends of the molecules turn them into electron conducting [157]. Such kind of substitution-induced carrier switching deserves much attention for possible use in electronic devices such as organic FETs [66].

\section{Photovoltaic activities of the acenes and heteroacenes}

Recently, a significant effort has been devoted to the rational design and synthesis of $\pi$-conjugated small organic molecules with the aim of their successful implementation in solar cell devices. These include different kinds of acenes, heteroacenes, etc. with a common molecular architecture D- $\pi$-A [20,57,58,85,95,96,161-164]. However, the reported power conversion efficiencies of the devices are relatively poor. This is because of the fact that in spite of suitable optical gap of the molecular dyes, the absorption coefficients for the low energy transitions are low. Secondly, those acene- and heteroacene-based dyes are relatively less soluble in water as well as in other common organic solvents. Furthermore, most of the acene-based dyes tend to aggregate which substantially reduces their power conversion efficiency [82,165]. Nevertheless, people have also found the ways to get rid of these problems, e.g., by functionalizing the acenes by various functional groups. Anthracene-based tetrathiafulvalene derivative (ext-TTF), in particular is very promising in this regard [165-168]. However, in the following sections, we will point out some interesting features of $\mathrm{PCn}$ and $\mathrm{SN} n$ molecules as potential donors in small molecule-based BHJ solar cells.

Before going to the details of such discussion, let us have a look on the controlling parameters of a photovoltaic cell. The efficiency of a BHJ solar cell can be expressed as

$$
\eta=\frac{\mathrm{FF} \cdot J_{\mathrm{SC}} \cdot V_{\mathrm{OC}}}{P_{\mathrm{inc}}},
$$

where FF is the fill factor at which the device operates with the maximum power. This is mainly related to the total series resistance of the cell. $P_{\text {inc }}$ is the input power of incident solar radiation and $J_{\mathrm{SC}}$ is the short circuit current density of photovoltaic cell which can be expressed as [169]

$$
J_{\mathrm{SC}}=\int \operatorname{LHE}(\lambda) \Phi_{\text {inject }} \eta_{\text {collect }} \mathrm{d} \lambda
$$

where $\operatorname{LHE}(\lambda)$, the light harvesting efficiency is a function of absorption wavelength $(\lambda)$, which can be evaluated from the intensity of absorption (oscillator strength, $f$ ) as given below:

$$
\operatorname{LHE}(\lambda)=1-10^{-f},
$$

and $\Phi_{\text {inject }}$ and $\eta_{\text {collect }}$ are the electron injection efficiency to conduction band of the A and the electron collection efficiency at the transparent conductive electrode, respectively. Note that $\Phi_{\text {inject }}$ is related to the driving force $\left(\Delta G_{\text {inject }}\right)$ of electrons injection from the photoexcited $\mathrm{D}$ to the A counterpart which can be approximated as [124,126]

$$
\Delta G_{\text {inject }}=E_{\mathrm{LUMO}}^{\mathrm{D}}-E_{\mathrm{LUMO}}^{\mathrm{A}} .
$$

Apart from these, the open-circuit voltage $\left(V_{\mathrm{OC}}\right)$ of a $\mathrm{BHJ}$ solar cell can be estimated as [170]

$$
V_{\mathrm{OC}}=\frac{1}{e}\left(\left|E_{\mathrm{HOMO}}^{\mathrm{D}}\right|-\left|E_{\mathrm{LUMO}}^{\mathrm{A}}\right|\right)-0.3 V,
$$

assuming that an energy difference of $0.3 \mathrm{eV}$ between the LUMOs of D and A is sufficient for efficient charge separation from the initially produced exciton.

\subsection{Pentacene and its derivatives}

The major characteristic of a donor molecule in photovoltaic cell is to produce suitable energy band alignment with a suitable acceptor counterpart. $\mathrm{PCn}-\mathrm{C}_{60}$ nanocomposites are well studied for this purpose $[83,84,121]$. Here, we explore the possibility of tuning frontier energy levels of PCn by substituting various functional groups. Noteworthily, the electron donating group in general pushes up both the energy levels of HOMO and LUMO, while the electron withdrawing group pulls down both of them. However, the extent of raising or lowering of the individual frontier orbitals may differ, resulting in slight change in the HOMO-LUMO gap. Table 2 depicts the relative energy positions of the HOMOs and LUMOs and their respective gaps for differently substituted PCn molecules. Figure 12 shows a more clear pictograph of their relative positions with respect to those of an acceptor, $\mathrm{C}_{60}$. From the relative energy positions, one may expect that all the donor molecules are capable of donating photoexcited electron from their respective LUMOs to the LUMO of $\mathrm{C}_{60}$. As a matter of fact, this energy difference between the LUMOs of D and A measures the driving force for electron injection $\left(\Delta G_{\text {inject }}\right)[126,171,172]$. Thus, one may choose suitable functionalized PCn molecules for modelling photovoltaic devices with better performances. Very strong electron donating group may increase the rate of electron injection, but this subsequently increases the position of the HOMO too which in turn increases the chance of electron-hole recombination, causing negative effect on photovoltaic performance. So, a balance is required between the charge injection and recombination rates [84].

To have a more realistic picture, one needs to study the D-A composite system which can mimic the fabricated device. The 
Table 2. Computed HOMO/LUMO energy levels ( $\left.E_{\text {HOMO/LUMO }}\right)$, their respective gaps $(\Delta)$, open circuit voltage $\left(V_{\mathrm{OC}}\right)$, free energy change for electron injection $\left(\Delta G_{\text {inject }}\right)$ and dipole moment $(\mu)$ for the $\mathrm{PCn}$ derivatives.

\begin{tabular}{lccccccc}
\hline Sl. no. & Species & $E_{\text {HOMO }}(\mathrm{eV})$ & $E_{\text {LUMO }}(\mathrm{eV})$ & $\Delta(\mathrm{eV})$ & $V_{\mathrm{OC}}(\mathrm{eV})$ & $\Delta G_{\text {inject }}(\mathrm{eV})$ & $\mu(\mathrm{D})$ \\
\hline I & PCn & -4.60 & -2.39 & 2.21 & 1.07 & 0.84 & 0.00 \\
II & PCn-DA & -4.82 & -2.73 & 2.09 & 1.29 & 0.50 & 0.00 \\
III & $\mathrm{NO}_{2}$-PCnA & -5.09 & -3.04 & 2.05 & 1.56 & 0.19 & 3.10 \\
IV & $\mathrm{CN}_{\text {PCnA }}$ & -5.07 & -3.05 & 2.03 & 1.54 & 0.18 & 3.36 \\
V & $\mathrm{NH}_{2}$-PCnA & -4.44 & -2.39 & 2.04 & 0.91 & 0.84 & 4.06 \\
VI & $\mathrm{OM}_{\text {MePnA }}$ & -4.66 & -2.54 & 2.12 & 1.13 & 0.69 & 2.19 \\
VII & $\mathrm{CO}_{2}$-PCnA & -4.86 & -2.77 & 2.09 & 1.33 & 0.46 & 0.58 \\
VIII & $\mathrm{CF}_{3}$-PCnA & -4.94 & -2.85 & 2.09 & 1.41 & 0.38 & 1.29 \\
IX & $\mathrm{CH}_{3}$-PCnA & -4.66 & -2.53 & 2.12 & 1.13 & 0.70 & 2.45 \\
& $\mathrm{C}_{60}$ & -5.98 & -3.23 & 2.76 & - & - & - \\
\hline
\end{tabular}

R-PCnA indicates the replacement of terminal carboxylic acid group of PCn-DA by the R group. The corresponding values of $\mathrm{C}_{60}$ are also given for comparison [84].

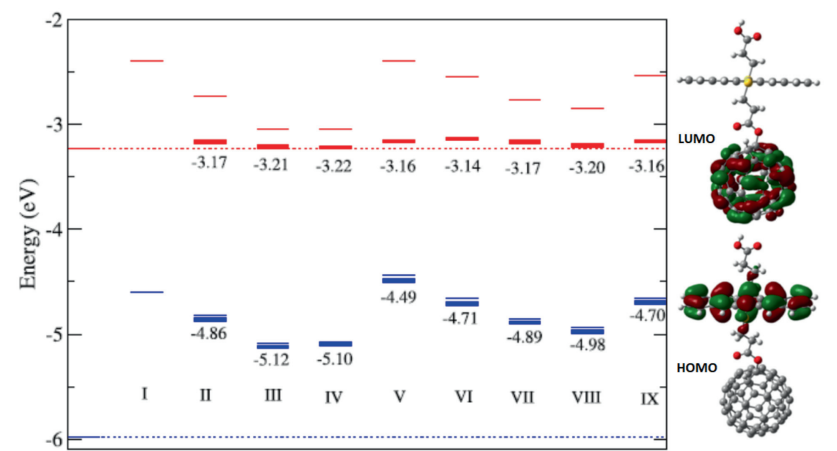

Figure 12. Relative energy positions of the HOMO (blue) and LUMO (red) states for the individual molecules (narrow line) and the corresponding composites with $\mathrm{C}_{60}$ (bold line) obtained at the B3LYP/6-31G(d) level of theory. The Roman numbers correspond to the molecules as those in table 2. Similar HOMO and LUMO energy levels of $\mathrm{C}_{60}$ are presented by dotted blue and red lines, respectively. $\mathrm{HOMO} / \mathrm{LUMO}$ isosurface plots of the PCn-DA- $\mathrm{C}_{60}$ composite are shown to indicate the spatial separation of electron and hole densities [84].

photovoltaic properties of such a model structure can actually predict the performance of such device. Even a computational analysis of such composite structure can provide intuitive ideas to further improve the power conversion efficiency. As found from table 2 and figure 12, the individual energy positions of the PCn derivatives and the $\mathrm{C}_{60}$ counterparts have been changed to some extent in the composite system due to D-A CT interactions. However, the relative energy ordering is retained, thereby giving rise to a type-II band alignment, which still indicates the possibility of charge injection from $\mathrm{D}$ to A counterpart. Molecular orbital analysis of the composite materials clearly reveals that the HOMO of the composite system always resides on the donor PCn derivative whereas the LUMO stays on the acceptor, $\mathrm{C}_{60}$. This special charge separation substantially reduces the chance of charge recombination $[84,111,112,171]$. A more clear picture could be figured out from the analysis of the corresponding electronically excited state of the composite system, namely the $\mathrm{CT}$ state which originates as a result of CT from the HOMO of the D to the LUMO of the A counterpart $[84,119,120,124]$.

In organic PVCs, the process of charge generation involves photoinduced electron transfer from D to A counterpart as already discussed. This leads to either a direct chargeseparated state or in most cases, an intermediate CT state is produced, which then evolves into a charge-separated state $[173,174]$. The CT state is thus a key intermediate electronic state in the operational process of organic photovoltaic devices because it plays the deterministic role in the exciton-dissociation as well as in the charge recombination processes $[121,175]$. Combined time-resolved femto-second spectroscopy and first-principles quantum dynamics simulation studies demonstrate how the coherent motion of atoms governs the photo-induced charge generation and thereafter interfacial charge separation, which generally occur at the femto-second (fs) time scale [176]. These studies also point out the precise role of vibronic coupling in the evolution of the CT state.

Combined $a b$ initio molecular dynamics (AIMD) simulations and DFT calculations have been performed to probe the dynamics of the lowest $\mathrm{CT}$ state of the $\mathrm{PCn}-\mathrm{C}_{60}$ composite at the fs time scale that furnishes better insights into the charge-separation and recombination dynamics at the D-A interface [121]. As could be visualized from the top panel of figure 13, the two lowest excited states of the PCn- $\mathrm{C}_{60}$ composite are identified as the $\mathrm{CT}$ states, while the third and fourth excited states are admixture of local excitations (LE) on $\mathrm{PCn}$ and a CT excitation [121]. The excess (negative) charges on $\mathrm{C}_{60}$ for the lowest four excited states are also displayed in the same figure. Importantly, the total energy difference between the first and fourth excited states is only in the order 


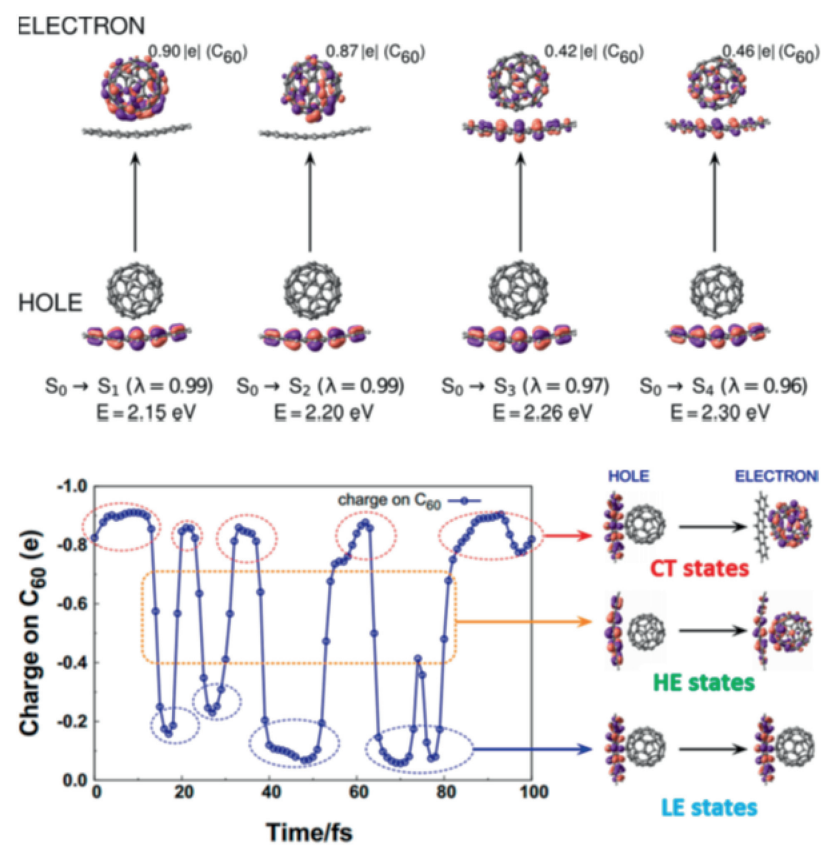

Figure 13. Top panel: the lowest four excited states of the $\mathrm{PCn}-\mathrm{C}_{60}$ composite. $\lambda$ indicates the contributory fraction of the hole-electron pair in the given electronic transition. The amount of Mulliken charge on $\mathrm{C}_{60}$ is also presented to indicate the extent of charge separation. Bottom panel: time-dependent variation of Mulliken charge population on $\mathrm{C}_{60}$ in the $\mathrm{PCn}-\mathrm{C}_{60}$ complex for a representative trajectory (Traj-3). Calculations are done at the tuned TD- $\omega$ B97XD/6-31G(d) $\left(\omega=0.137 \mathrm{bohr}^{-1}\right)$ level of theory [121].

of $150 \mathrm{meV}$. This actually makes the composite particularly interesting because it can be taken as a representative of $\mathrm{D}-\mathrm{A}$ composite systems having a small energy gap between the LE and CT states which is highly desired to minimize the energy (and voltage) loss during the energy conversion processes.

The dynamics of the lowest excited state could be followed to see the electron-phonon coupling involving some high-frequency modes within the PCn molecule. Usually, the $\mathrm{C}-\mathrm{C}$ bond-stretching and ring-breathing vibrational modes strongly couple with the electronic excitations in such $\pi$ conjugated molecules. This typically happens within the 100 fs time scale [121]. Here, a useful descriptor of the dynamics in the first excited state $\left(S_{1}\right)$ is the amount of excess negative charge on the A counterpart $\left(\mathrm{C}_{60}\right)$. The time evolution of such state is shown in the bottom panel of figure 13 . Noteworthily, during the course of the CT processes, the hole wave function in the $S_{1}$ state remains localized on A, while the electron wave function strongly fluctuates between the $\mathrm{D}$ (PCn) and $\mathrm{A}$. The electron starts on $\mathrm{C}_{60}$, jumps onto the $\mathrm{PCn}$ molecule after some 18-20 fs of time before going back to $\mathrm{C}_{60}$. In the meantime, it takes $2-3 \mathrm{fs}$ of time to complete a jump, in the middle of which the $\mathrm{S}_{1}$ state has a hybrid LECT character. However, as a whole, it can be argued that the electron transfer between $\mathrm{PCn}$ and $\mathrm{C}_{60}$ occurs within a fs time scale $[121,177,178]$.



Figure 14. Time-dependent evolution of the LUMO, LUMO+1, LUMO+2 and LUMO+3 energy levels of the PCn- $\mathrm{C}_{60}$ complex for the Traj-3 trajectory, computed by using the $\omega \mathrm{B} 97 \mathrm{XD} / 6-31 \mathrm{G}(\mathrm{d})$ $\left(\omega=0.137\right.$ bohr $\left.^{-1}\right)$ level of theory [121].

For better understanding the oscillations in the electron transfer processes between $\mathrm{PCn}$ and $\mathrm{C}_{60}$, one may analyse the fluctuations in the $\mathrm{MO}$ energies of the $\mathrm{PCn}-\mathrm{C}_{60}$ composite. The HOMO and LUMO+3 levels of the composite are shown to reside on PCn, while the LUMO, LUMO+1 and LUMO+2 levels (localized on the $\mathrm{C}_{60}$ ) are nearly degenerate and correspond to the triply degenerate LUMO states of $\mathrm{C}_{60}$ [121]. From figure 14 , it is clearly revealed that when $\mathrm{S}_{1}$ state of the composite gains a LE character, the (PCnbased) LUMO+3 energy comes close to the $\left(\mathrm{C}_{60}\right.$-based) LUMO+2 energy. Interestingly, the transition between the LE and CT characters of the $S_{1}$ state passes through a mixed LE-CT state as already discussed. Such a transient hybrid state has recently been observed in a polymer- $\mathrm{C}_{60^{-}}$ based thin film solar cells [179]. Noteworthily, in that case also, the energy difference between the $S_{1}$ state (polymerbased) and the interfacial $\mathrm{CT}$ state is reported to be very small.

\subsection{S,N-heteroacenes}

We already introduced that even $\mathrm{SN} n$ molecules experience poorer charge separation in their first excited state in comparison with that of the odd ones which should be reflected in their photovoltaic activities in D-A type of composites [85,93]. Before going to such details, let us have a look on the absorption and excited state properties of DCV substituted SN5 and SN6. On the basis of first principle calculations, it has been reported that the first excitation energy, which is designated as the band-gap absorption, decreases by $0.07 \mathrm{eV}$, while going from SN5 to SN6 [85]. Although for both the molecules, the excited-state CT amount $\left(q^{\mathrm{CT}}\right)$ is almost same, the associated CT lengths $\left(l^{\mathrm{CT}}\right)$ show a remarkable difference. For the first excited state, the computed $l^{\mathrm{CT}}$ values are reported to be 0.068 and $0.0001 \AA$ for SN5 and SN6, respectively. This affects the charge transport and carrier dynamic seriously. Additionally, because of their rigid coplanar backbone, the $\mathrm{SN} n$ molecules show intense photoluminescence and high carrier 


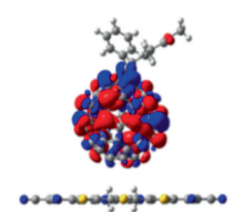

(a)

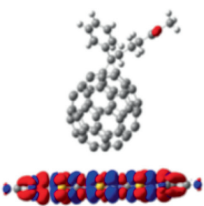

(b)

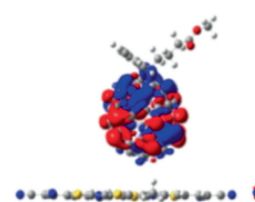

(d)

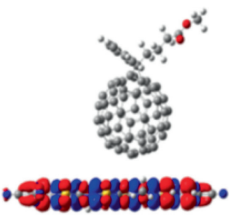

(e)

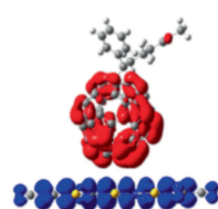

(c)

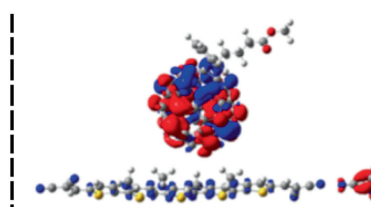

(a)

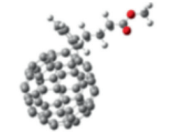

(b)

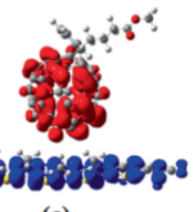

(c)



(f)



Figure 15. Left panel: (a-c) CDD plots for $\mathrm{S}_{1}(\mathrm{LE}), \mathrm{S}_{5}$ (LE) and $\mathrm{S}_{14}(\mathrm{CT})$ excited states of the SN5-PC 61 BM composite system, and (d-f) $\mathrm{S}_{1}$ (LE), $\mathrm{S}_{5}$ (LE) and $\mathrm{S}_{18}$ (CT) excited states of the SN6-PC 61 BM composite system. Right panel: (a-c) CDD plots for $\mathrm{S}_{1}$ (LE), $\mathrm{S}_{3}$ (LE) and $\mathrm{S}_{5}(\mathrm{CT})$ excited states of the SN9-PC 61 BM composite system, and (d-f) $\mathrm{S}_{1}$ (LE), $\mathrm{S}_{3}$ (LE) and $\mathrm{S}_{6}$ (CT) excited states of the SN10-PC 61 BM composite system. LE and CT stand for local excitations (involving either $\mathrm{SN} n$ or $\mathrm{PC}_{61} \mathrm{BM}$ ) and CT states, respectively. Blue (red) stands for depletion (accumulation) of negative charges [85].

mobilities [93,180]. The coplanar architecture of the molecules also suppresses the conformational disorder, resulting in a decrease in reorganization energy which in turn increases their intrinsic charge mobility and hence photocurrent efficiency (PCE) [91]. The planar mixed active layer of DCV-SN5 blended with $\mathrm{C}_{70}$ is reported to show an excellent PCE of $5.64 \%$, while under similar conditions, SN6 derivative shows only 3.02\% PCE [93]. In sharp contrast, the absorption intensity of the first intense transition for SN6 is higher than that of SN5. Furthermore, some subtransition dipoles are eliminated due to $C_{2 \mathrm{~V}}$ symmetry of the SN5. So, in spite of better absorption characteristics, the improper charge separation leads to faster charge recombination and thus, the PCE is lower for SN6 [85,93]. For its better justification and to generalize such phenomena, a recent computational work has been performed on an additional pair of SN9 and SN10 which also reports similar observation [85].

In view of their practical implementation, a more realistic model, D-A type of composites have been studied with different $\mathrm{SN} n$ molecules as $\mathrm{D}$ and $\mathrm{PC}_{61} \mathrm{BM}$ as an $\mathrm{A}$. In the optimized structure, the $\mathrm{PC}_{61} \mathrm{BM}$ occupies a vertical orientation over the $\mathrm{SN} n$ molecular surface. The computed optical properties reveal that all the low-energy transitions are of LE type involving either the $\mathrm{D}$ or the A counterpart. The first CT state for SN5- $\mathrm{PC}_{61} \mathrm{BM}$ is characterized to be $\mathrm{S}_{14}$ (appearing at $403 \mathrm{~nm}$ ), while for the SN6-composite is $S_{18}$. Although the absorption intensities of the transitions relating to those states are poor, those states are mainly responsible for photovoltaic activities as already discussed [121]. The CT characteristics in those excited states could be visualized from the charge density difference (CDD) [181] plots as depicted in figure 15. Note that the CDD between the excited state and the corresponding ground state has been expressed in terms of $\Delta \rho(r)=\rho^{\text {elec }}(r)-\rho^{\text {hole }}(r)$, where the electron and hole densities are expressed in terms of the coefficients of configuration state function $\left(c_{i}\right)$ as [182]

$$
\begin{aligned}
\rho^{\mathrm{elec}}(r)= & \sum_{i \rightarrow l}\left(c_{i}^{l}\right)^{2} \phi_{i}(r) \phi_{i}(r) \\
& +\sum_{i \rightarrow l} \sum_{j \neq i \rightarrow l} c_{i}^{l} c_{j}^{l} \phi_{i}(r) \phi_{j}(r),
\end{aligned}
$$

and

$$
\begin{aligned}
\rho^{\text {hole }}(r)= & \sum_{i \rightarrow l}\left(c_{i}^{l}\right)^{2} \phi_{l}(r) \phi_{l}(r) \\
& +\sum_{i \rightarrow l} \sum_{i \rightarrow m \neq l} c_{i}^{l} c_{j}^{l} \phi_{l}(r) \phi_{m}(r) .
\end{aligned}
$$

Obviously, the CT states experience a huge CT length $\left(l^{\mathrm{CT}}\right)$ which also follows a similar trend as that for single $\mathrm{SN} n$ molecules. Similar is the situation for the SN9 and SN10 composites, for which the lowest $\mathrm{CT}$ states are $\mathrm{S}_{5}$ and $\mathrm{S}_{6}$, respectively (see figure 15). Note that much lower excitons are now responsible for generating the CT states.

The photovoltaic performance of a material is crucially determined by the carrier transfer dynamics within the system. In a simplified form, the corresponding rates of $\mathrm{CT}$ and recombination can be followed by using Marcus theory [127] as given below:

$$
k=\sqrt{\frac{4 \pi^{3}}{h^{2} \lambda k_{\mathrm{B}} T}}\left|V_{\mathrm{DA}}\right|^{2} \exp \left(-\frac{(\Delta G+\lambda)^{2}}{4 \lambda k_{\mathrm{B}} T}\right),
$$

where $\lambda$ is the reorganization energy, $V_{\mathrm{DA}}$ is the CT integral between the $\mathrm{D}$ and $\mathrm{A}$ states, and $\Delta G$ is the free energy change for the corresponding process $\left(\Delta G_{\mathrm{CT}}\right.$ for CT and 
Table 3. Computed charge transfer/recombination rate constants and associated dynamical parameters for the $\mathrm{SN} n-\mathrm{PC} 61 \mathrm{BM} / \mathrm{PDI}$ composite systems.

\begin{tabular}{lccccccc}
\hline System & $E_{\mathrm{b}}(\mathrm{eV})$ & $\Delta \mu$ (a.u.) & $\mu_{\mathrm{tr}}($ a.u. $)$ & $V_{\mathrm{DA}}(\mathrm{eV})$ & $V_{\mathrm{OC}}(\mathrm{V})$ & $\Delta G_{\mathrm{CR}}$ in eV $\left(k_{\mathrm{CR}}\right.$ in s & $\Delta G_{\mathrm{CT}}$ in eV $\left(k_{\mathrm{CT}}\right.$ in s \\
\hline SN5-PC 61 BM & 0.35 & 3.06 & 0.0355 & 0.0357 & 1.88 & $-2.18\left(4.11 \times 10^{-11}\right)$ & $-0.58\left(2.67 \times 10^{13}\right)$ \\
SN6-PC 61 BM & 0.31 & 6.92 & 0.0079 & 0.0036 & 1.81 & $-2.11\left(4.60 \times 10^{-11}\right)$ & $-0.53\left(3.10 \times 10^{11}\right)$ \\
SN9-PC 61 BM & 0.26 & 4.17 & 0.4726 & 0.2745 & 1.29 & $-1.59\left(1.68 \times 10^{5}\right)$ & $-0.72\left(6.97 \times 10^{14}\right)$ \\
SN10-PC 61 BM & 0.23 & 7.98 & 0.1359 & 0.0451 & 1.28 & $-1.58\left(7.56 \times 10^{3}\right)$ & $-0.67\left(2.70 \times 10^{13}\right)$ \\
SN5-PDI & 0.35 & 5.47 & 0.1518 & 0.0686 & 1.29 & $-1.59\left(1.01 \times 10^{4}\right)$ & $-1.17\left(1.85 \times 10^{10}\right)$ \\
SN9-PDI & 0.26 & 6.60 & 0.0250 & 0.0089 & 0.70 & $-1.00\left(1.40 \times 10^{10}\right)$ & $-1.31\left(5.92 \times 10^{6}\right)$ \\
\hline
\end{tabular}

Calculations are performed in the gas phase. $E_{\mathrm{b}}, \Delta \mu, \mu_{\mathrm{tr}}$ and $V_{\mathrm{OC}}$ stand for exciton binding energy, dipole moment difference between ground and excited states, transition dipole between ground and excited states, and open-circuit voltage, respectively [85].

$\Delta G_{\mathrm{CR}}$ for charge recombination) at a particular temperature $T$. In principle, $\Delta G_{\mathrm{CR}}=E_{\mathrm{IP}}(\mathrm{D})-E_{\mathrm{EA}}(\mathrm{A})$, where $E_{\mathrm{IP}}(\mathrm{D})$ and $E_{\mathrm{EA}}(\mathrm{A})$ are the ionization potential of $\mathrm{D}$ and electron affinity of $\mathrm{A}$, respectively. However, $E_{\mathrm{IP}}(\mathrm{D})$ and $E_{\mathrm{EA}}(A)$ could approximately be estimated as the HOMO and LUMO energies of the D and A counterparts, respectively [183]. Now, with the help of $\Delta G_{\mathrm{CR}}$, one can estimate the value of $\Delta G_{\mathrm{CT}}$ using the Rehm-Weller equation [184], $\Delta G_{\mathrm{CT}}=$ $-\Delta G_{\mathrm{CR}}-\Delta E_{0-0}-E_{\mathrm{b}}$; where $\Delta E_{0-0}$ and $E_{\mathrm{b}}$ are the first excitation energy of the free-base donor and the corresponding exciton binding energy [185], respectively.

Equation (10) involves another important parameter i.e., reorganization energy $(\lambda)$ which basically consists of two parts, inner reorganization energy $\left(\lambda_{\mathrm{i}}\right)$ and outer reorganization energy $\left(\lambda_{s}\right)$ [120]. $\lambda_{i}$ is originated from the geometric change in the D and A counterparts of the composite at the time of charge separation phenomena and thus, it is the sum of two terms, electron and hole reorganization energies $\left(\lambda_{\mathrm{e}}\right.$ and $\lambda_{\mathrm{h}}$, respectively). On the other hand, the $\lambda_{\mathrm{s}}$ comes from the electronic and nuclear polarizations/relaxations of the surrounding medium. Now, $\lambda_{\mathrm{e}}$ and $\lambda_{\mathrm{h}}$ could be estimated as $\lambda_{\mathrm{e}}=E\left(\mathrm{~A}^{-}\right)-E(\mathrm{~A})$ and $\lambda_{\mathrm{h}}=E(\mathrm{D})-E\left(\mathrm{D}^{+}\right)$, where $E\left(\mathrm{~A}^{-}\right)$and $E(\mathrm{~A})$ are the energies of the neutral acceptor at the anionic geometry and optimal ground-state geometry, respectively; and $E(\mathrm{D})$ and $E\left(\mathrm{D}^{+}\right)$are the energies of the radical cation donor at the neutral geometry and optimal cation geometry, respectively. In an alternative way, $\lambda_{\mathrm{i}}$ can be calculated by taking the average of $\lambda_{\mathrm{i} 1}$ and $\lambda_{\mathrm{i} 2}$, where the two terms come from the reorganization of intramolecular excited state to charge separated state and vice versa, respectively $[119,186]$. However, the theoretically calculated $\lambda_{\mathrm{i}}$ values of the dye- $-\mathrm{PC}_{61} \mathrm{BM}$ composites run in the range of $0.20-0.25 \mathrm{eV}$ $[85,124,187]$. The estimated values of $\lambda_{\mathrm{s}}$ also lie in that region such that the overall $\lambda$ values appear around $0.50 \mathrm{eV}$ which corroborate well with the experimental values $[124,188]$.

Now, the D-A CT integral $\left(V_{\mathrm{DA}}\right)$ for the CT state involving the transition from the ground state $\left(\mathrm{S}_{0}\right)$ to the excited state $\left(\mathrm{S}_{n}\right)$ can be expressed in terms of generalized Mulliken-Hush $(\mathrm{GMH})$ model [189]

$$
V_{\mathrm{DA}}=\frac{\mu_{\mathrm{tr}} \Delta E}{\sqrt{(\Delta \mu)^{2}+4\left(\mu_{\mathrm{tr}}\right)^{2}}},
$$

where $\mu_{\mathrm{tr}}$ is the component of the transition dipole moment along the major axis, $\Delta \mu$ the dipole moment difference between the $\mathrm{S}_{0}$ and $\mathrm{S}_{n}$ states, and $\Delta E$ the vertical excitation energy. Now, $\Delta \mu$ can be computed by using the finite field method on the excitation energy [190,191]. The calculated $\Delta \mu, \mu_{\mathrm{tr}}$ and thereafter, the corresponding $V_{\mathrm{DA}}$ values for some $\mathrm{SN} n$-based composite systems are shown in table 3 .

As table 3 shows, for the SN5- $\mathrm{PC}_{61} \mathrm{BM}$ composite, the estimated value of $\Delta G_{\mathrm{CR}}$ is $-2.18 \mathrm{eV}$ and hence $\Delta G_{\mathrm{CT}}=$ $-0.58 \mathrm{eV}$. The negative value of $\Delta G_{\mathrm{CT}}$ indicates the thermodynamic feasibility of the electron transfer process. However, comparing the calculated rate constants of the concerned processes one may argue that SN5 involves a slower charge recombination and faster $\mathrm{CT}$ rate in comparison with those of SN6. Similarly, SN9 composite also experiences a faster $\mathrm{CT}$ rate than that of SN10. Therefore, it rationalizes the fact why the odd $\mathrm{SN} n$ molecules (SN5/SN9) provide better photovoltaic performances than the even ones [85,93]. A similar conclusion could also be drawn based on the composites comprising $\mathrm{SN} n$ and helical perylene diimide dimer (PDI) [192] as a non-fullerene acceptor [85].

\section{Conclusions and future outlook}

In summary, we present here, the very recent developments on the charge transport and CT properties of different nanojunctions based on PCn and $\mathrm{SN} n$ molecules of different conjugation lengths. In case of $\mathrm{PCn}$-graphene nanojunctions, the low bias conductance is mainly mediated by edge-induced transmission channels originating from zigzag edges of GNR. Attachment of strong electron withdrawing groups like $\mathrm{CN}$ to the PCn molecule will pull down the transmission eigenchannels closer to the Fermi level which increases the intensity of edge-induced transmittance, thereby increasing the conductivity. Proper functionalization of $\mathrm{PCn}$ with electron donating and withdrawing groups imparts an internal field across the molecule which subsequently lifts the degeneracy of the zGNR edge states and this is responsible for generating spin currents in the PCn-zGNR nanojunctions. Spin-dependent $I-V$ characteristics of these devices show NDR behaviour which is attributed to the poor coupling between the discrete 
molecular states of PCn and the zGNR leads at some specific bias region. Although the nanojunctions are predicted to show low spin filtering ability, the exhibited spin transport properties are quite useful for fabricating bias dependent spintronic devices.

The transport properties of different edge-terminated $\mathrm{SN} n$ molecule-gold-surface nanojunctions also deserve much attention. Although such nanojunctions constituting bare or $\mathrm{SMe}$-terminated $\mathrm{SN} n$ are hole conducting, termination with very strong electron withdrawing DCV groups led them to electron conducting. Such kind of SN $n$ molecule-based ntype materials are predicted to be thermodynamically stable and thus expected to be realized in near future. Very strong electron withdrawing effect of DCV pull down the molecular LUMO level, thereby promoting easier way of electron conduction through that state. Such kind of carrier switching through simple chemical modification on $\mathrm{SN} n$ would be very beneficial in OFET or diode industry.

On the other hand, by structural modification of PCn molecule through proper substitution, one can optimize the photovoltaic performance of a $\mathrm{BHJ}$ solar cell with $\mathrm{C}_{60}$ as an acceptor. It has been proposed that $\mathrm{PCn}$ molecules functionalized by strong electron donating substituents may offer a faster interfacial electron transfer rate, however, strong electron withdrawing groups like $\mathrm{CN}$ provide a better light harvesting efficiency. The electronic structure calculations reveal that $\mathrm{PCn}-\mathrm{C}_{60}$ nanocomposites show type-II band alignment which substantially reduces the charge recombination rates, thereby increasing photovoltaic performances. A combined DFT and AIMD simulations of the lowest excited state in the $\mathrm{PCn}-\mathrm{C}_{60}$ composite underline that some specific high frequency vibrational modes, namely the $\mathrm{C}-\mathrm{C}$ bond-stretching and ring-breathing in $\mathrm{PCn}$ are mainly responsible for the major oscillations in the energy levels of the composite. Thus, such vibronic couplings have an influence on the evolution of the lowest excited state of the composite which in fact activate transition between a CT and LE-type of states, via a transient hybrid LE-CT state. The dynamics in the composite system results in a distribution of the lowest excited-state energy over several tenths of eV. Such kind of phenomena has important implications regarding small molecule-based organic solar cells.

Recent experimental and quantum chemical investigations highlight the potential applicability of $\mathrm{SN} n$ molecules in photovoltaic devices. The rigid architecture of the $\mathrm{SN} n$ suppresses the conformation disorder and thus reduces the reorganization energy, thereby enhancing the intrinsic charge mobility. The first optical absorption energy gradually decreases with increasing the conjugation length of the $\mathrm{SN} n$ backbone. Additionally, strong electron withdrawing groups attached to the both ends of the $\mathrm{SN} n$ impart extended delocalization which results in further red-shifting in the absorption maxima. An even-odd relationship is observed for the intramolecular charge separation process of the $\mathrm{SN} n$, the odd ones providing longer CT length than the even ones. Similar event also happens at the $\mathrm{D}-\mathrm{A}$ interface with $\mathrm{PC}_{61} \mathrm{BM}$ as a global acceptor.
Thus, it has been predicted that the odd $\mathrm{SN} n$ molecules would provide a faster interfacial CT rate, indicating their better photovoltaic performances.

Acenes and heteroacenes are therefore very promising for the next generation optoelectronic devices. Their intrinsic molecular properties are very suitable for their extraordinary performances in charge transporting or energy conversion processes. The major drawback is their poor solubility in common solvents. Further studies are therefore necessary with the aim to increase their solubility through proper substitution, specially by polar electron withdrawing groups. However, this should not affect the carrier mobility too much, and secondly, the planar backbone architecture should also be retained. Finally, carrier transport dynamics is an essential tool for predicting the actual device performances of those materials. For that, one needs to study a large scale non-adiabatic molecular dynamics simulations over the D-A interface $[193,194]$. Although some efforts have already been given [193], we do hope that more sophisticated experimental and theoretical researches will bring further insights into the carrier transport dynamics of acenes and heteroacenes systems.

\section{Acknowledgements}

This work was supported by the financial grant of DST Nano Mission Project (ref. no. SR/NM/NS-1005/2016) provided by the Govt. of India. We sincerely acknowledge financial support from CSIR, New Delhi, Govt. of India (file no. 01(2916)/17/EMR-II). SB is grateful to CSIR for providing him with a Senior Research Fellowship.

\section{References}

[1] Duan C, Zhang K, Zhong C, Huang F and Cao Y 2013 Chem. Soc. Rev. 429071

[2] Beaujuge P M and Fréchet J M 2011 J. Am. Chem. Soc. 133 20009

[3] Facchetti A 2010 Chem. Mater. 23733

[4] Wang C, Dong H, Hu W, Liu Y and Zhu D 2011 Chem. Rev. 1122208

[5] Tsai C H, Chirdon D N, Maurer A B, Bernhard S and Noonan K J 2013 Org. Lett. 155230

[6] Shinar J and Savvateev V 2004 Introduction to organic lightemitting devices (New York: Springer)

[7] Mei J, Diao Y, Appleton A L, Fang L and Bao Z 2013 J. Am. Chem. Soc. 1356724

[8] Watanabe M, Chang Y J, Liu S W, Chao T H, Goto K, Islam M M et al 2012 Nat. Chem. 4574

[9] Anthony J E 2006 Chem. Rev. 1065028

[10] Goldmann C, Haas S, Krellner C, Pernstich K, Gundlach D and Batlogg B 2004 J. Appl. Phys. 962080

[11] Pramanik A, Mandal B, Sarkar S and Sarkar P 2014 Chem. Phys. Lett. 5971

[12] Lin Y Y, Gundlach D, Nelson S F and Jackson T N 1997 IEEE Trans. Electron Dev. 441325 
[13] Nelson S, Lin Y Y, Gundlach D and Jackson T 1998 Appl. Phys. Lett. 721854

[14] Dimitrakopoulos C D and Malenfant P R 2002 Adv. Mater. 1499

[15] Chason M, Brazis P W, Zhang J, Kalyanasundaram K and Gamota D R 2005 Proc IEEE 931348

[16] Yang H, Shin T J, Ling M M, Cho K, Ryu C Y and Bao Z 2005 J. Am. Chem. Soc. 12711542

[17] Zu Heringdorf F J M, Reuter M and Tromp R 2001 Nature 412517

[18] Wen S H, Deng W Q and Han K L 2010 Phys. Chem. Chem. Phys. 129267

[19] Pshenichnyuk I A, Coto P B, Leitherer S and Thoss M 2013 J. Phys. Chem. Lett. 4809

[20] Kast H, Mishra A, Schulz G L, Urdanpilleta M, Mena-Osteritz E and Bäuerle P 2015 Adv. Funct. Mater. 253414

[21] Kihira Y, Shimada T, Matsuo Y, Nakamura E and Hasegawa T 2009 Nano Lett. 91442

[22] Kim W K and Lee J L 2006 Appl. Phys. Lett. 88262102

[23] Ihm K, Kim B, Kang T H, Kim K J, Joo M H, Kim T H et al 2006 Appl. Phys. Lett. 89033504

[24] Amy F, Chan C and Kahn A 2005 Org. Electron. 685

[25] Han M Y, Özyilmaz B, Zhang Y and Kim P 2007 Phys. Rev. Lett. 98206805

[26] Chen Z, Lin Y M, Rooks M J and Avouris P 2007 Physica E 40228

[27] Li X, Wang X, Zhang L, Lee S and Dai H 2008 Science 319 1229

[28] Jin C, Lan H, Peng L, Suenaga K and Iijima S 2009 Phys. Rev. Lett. 102205501

[29] Cao Y, Liu S, Shen Q, Yan K, Li P, Xu J et al 2009 Adv. Funct. Mater. 192743

[30] Prins F, Monrabal-Capilla M, Osorio E A, Coronado E and van der Zant H S 2011 Adv. Mater. 231545

[31] Di C A, Wei D, Yu G, Liu Y, Guo Y and Zhu D 2008 Adv. Mater. 203289

[32] Lee C G, Park S, Ruoff R S and Dodabalapur A 2009 Appl. Phys. Lett. 95188

[33] Liu W, Jackson B L, Zhu J, Miao C Q, Chung C H, Park Y J et al 2010 ACS Nano 43927

[34] Wakabayashi K 2001 Phys. Rev. B 64125428

[35] Sasaki K I, Murakami S and Saito R 2006 Appl. Phys. Lett. 88113110

[36] Son Y W, Cohen M L and Louie S G 2006 Nature 444347

[37] Son Y W, Cohen M L and Louie S G 2006 Phys. Rev. Lett. 97216803

[38] Hod O, Barone V, Peralta J E and Scuseria G E 2007 Nano Lett. 72295

[39] Rudberg E, Sałek P and Luo Y 2007 Nano Lett. 72211

[40] Nakada K, Fujita M, Dresselhaus G and Dresselhaus M S 1996 Phys. Rev. B 5417954

[41] Joly V J, Kiguchi M, Hao S J, Takai K, Enoki T, Sumii R et al 2010 Phys. Rev. B $\mathbf{8 1} 245428$

[42] Topsakal M, Sevinçli H and Ciraci S 2008 Appl. Phys. Lett. 92173118

[43] Zheng J, Yan X, Yu L, Li H, Qin R, Luo G et al 2011 J. Phys. Chem. $C 1158547$

[44] Kang J, Wu F and Li J 2011 Appl. Phys. Lett. 98083109

[45] Zeng H, Zhao J, Wei J and Hu H 2011 Eur. Phys. J. B 79335

[46] Zeng M, Shen L, Yang M, Zhang C and Feng Y 2011 Appl. Phys. Lett. 98053101
[47] Mandal B, Sarkar S, Pramanik A and Sarkar P 2013 J. Appl. Phys. 114173701

[48] Sarkar S, Pramanik A and Sarkar P 2016 Chem. Phys. 478 173

[49] Mandal B, Sarkar S, Pramanik A and Sarkar P 2013 Phys. Chem. Chem. Phys. 1521001

[50] Mandal B, Sarkar S, Pramanik A and Sarkar P 2012 J. Appl. Phys. 112113710

[51] Pramanik A, Sarkar S and Sarkar P 2012 J. Phys. Chem. C 11618064

[52] Modarresi M, Roknabadi M and Shahtahmasbi N 2011 Physica $E \mathbf{4 3} 1751$

[53] Saffarzadeh A and Farghadan R 2011 Appl. Phys. Lett. 98 023106

[54] Song Y, Xie Z, Ma Y, Li Z and Wang C K 2014 J. Phys. Chem. C 11818713

[55] Kang H S and Pramanik A 2011 J. Chem. Phys. 135124708

[56] Mandal B, Sarkar S, Pramanik A and Sarkar P 2014 RSC Adv. 449946

[57] Mishra A, Popovic D, Vogt A, Kast H, Leitner T, Walzer K et al 2014 Adv. Mater. 267217

[58] Qin P, Kast H, Nazeeruddin M K, Zakeeruddin S M, Mishra A, Bäuerle P et al 2014 Energy Environ. Sci. 72981

[59] Wetzel C, Mishra A, Mena-Osteritz E, Liess A, Stolte M, Würthner F et al 2013 Org. Lett. 16362

[60] Wetzel C, Brier E, Vogt A, Mishra A, Mena-Osteritz E and Bäuerle P 2015 Angew. Chem. Int. Ed. 5412334

[61] Aragó J, Viruela P M, Ortí E, Malavé Osuna R, Vercelli B, Zotti G et al 2010 Chemistry 165481

[62] Osaka I, Zhang R, Sauvé G, Smilgies D M, Kowalewski T and McCullough R D $2009 \mathrm{~J}$. Am. Chem. Soc. 131 2521

[63] Quinn J T, Zhu J, Li X, Wang J and Li Y 2017 J. Mater. Chem. C 58654

[64] Zaumseil J and Sirringhaus H 2007 Chem. Rev. 1071296

[65] Yan H, Chen Z, Zheng Y, Newman C, Quinn J R, Dötz F et al 2009 Nature 457679

[66] Dhondge A P, Chen J Y, Lin T, Yen F M, Li K W, Hsieh H C et al 2018 Org. Lett. 2040

[67] Cochran J E, Amir E, Sivanandan K, Ku S Y, Seo J H, Collins B A et al 2013 J. Polym. Sci. B: Polym. Phys. 5148

[68] Facchetti A, Yoon M H, Stern C L, Katz H E and Marks T J 2003 Angew. Chem. Int. Ed. 423900

[69] Pramanik A and Sarkar P 2015 Phys. Chem. Chem. Phys. 17 26703

[70] Gryn'ova G, Ollitrault P J and Corminboeuf C 2017 Phys. Chem. Chem. Phys. 1923254

[71] Ando S, Nishida J I, Fujiwara E, Tada H, Inoue Y, Tokito S et al 2005 Chem. Mater. 171261

[72] Melucci M, Frere P, Allain M, Levillain E, Barbarella G and Roncali J 2007 Tetrahedron 639774

[73] Su T A, Li H, Steigerwald M L, Venkataraman L and Nuckolls C 2015 Nat. Chem. 7215

[74] Pramanik A and Sarkar P 2015 J. Chem. Phys. 143114314

[75] Dell E J and Campos L M 2012 J. Mater. Chem. 2212945

[76] Dell E J, Capozzi B, Xia J, Venkataraman L and Campos L M 2015 Nat. Chem. 7209

[77] Capozzi B, Xia J, Adak O, Dell E J, Liu Z F, Taylor J C et al 2015 Nat. Nanotechnol. 10522

[78] Tang M L, Reichardt A D, Wei P and Bao Z 2009 J. Am. Chem. Soc. 1315264 
[79] Simbeck A J, Qian G, Nayak S K, Wang G C and Lewis K M 2012 Surf. Sci. 6061412

[80] Xu B and Tao N J 2003 Science 3011221

[81] Blum A S, Yang J C, Shashidhar R and Ratna B 2003 Appl. Phys. Lett. 823322

[82] Pramanik C, Li Y, Singh A, Lin W, Hodgson J L, Briggs J B et al 2013 J. Mater. Chem. C 12193

[83] Yoo S, Domercq B and Kippelen B 2004 Appl. Phys. Lett. 85 5427

[84] Pramanik A, Sarkar S, Pal S and Sarkar P 2015 Phys. Lett. A 3791036

[85] Biswas S, Pramanik A, Pal S and Sarkar P 2017 J. Phys. Chem. C 1212574

[86] Ostroverkhova O, Shcherbyna S, Cooke D G, Egerton R F, Hegmann F A, Tykwinski R R et al 2005 J. Appl. Phys. 98 033701

[87] Mishra A and Bäuerle P 2012 Angew. Chem. Int. Ed. 512020

[88] Malytskyi V, Simon J J, Patrone L and Raimundo J M 2015 RSC Adv. 5354

[89] Fitzner R, Mena-Osteritz E, Mishra A, Schulz G, Reinold E, Weil M et al 2012 J. Am. Chem. Soc. 13411064

[90] Chen Y, Wan X and Long G 2013 Acc. Chem. Res. 462645

[91] Wu J S, Cheng Y J, Lin T Y, Chang C Y, Shih P I and Hsu C S 2012 Adv. Funct. Mater. 221711

[92] Mitsudo K, Shimohara S, Mizoguchi J, Mandai H and Suga S 2012 Org. Lett. 142702

[93] Chung C L, Chen C Y, Kang H W, Lin H W, Tsai W L, Hsu C C et al 2016 Org. Electron. 28229

[94] Chung C L, Chen C H, Tsai C H and Wong K T 2015 Org. Electron. 188

[95] Lee H, Jo H, Kim D, Biswas S, Sharma G D and Ko J 2016 Dyes Pigm. 129209

[96] Wetzel C, Mishra A, Mena-Osteritz E, Walzer K, Pfeiffer M and Bäuerle P 2016 J. Mater. Chem. C 43715

[97] O'regan B and Grätzel M 1991 Nature 353737

[98] Tachibana Y, Hara K, Sayama K and Arakawa H 2002 Chem. Mater. 142527

[99] Rehm J M, McLendon G L, Nagasawa Y, Yoshihara K, Moser J and Grätzel M 1996 J. Phys. Chem. 1009577

[100] Kitamura T, Ikeda M, Shigaki K, Inoue T, Anderson N A, Ai X et al 2004 Chem. Mater. 161806

[101] Anselmi C, Mosconi E, Pastore M, Ronca E and De Angelis F 2012 Phys. Chem. Chem. Phys. 1415963

[102] Oprea C I, Panait P, Cimpoesu F, Ferbinteanu M and Gîrţu M A 2013 Materials 62372

[103] Ronca E, Pastore M, Belpassi L, Tarantelli F and De Angelis F 2013 Energy Environ. Sci. 6183

[104] Zhang L, Cole J M and Dai C 2014 ACS Appl. Mater. Interfaces 67535

[105] Pratik S M and Datta A 2013 Phys. Chem. Chem. Phys. 15 18471

[106] Biswas S, Pramanik A and Sarkar P 2017. Comput. Theor. Chem. 110338

[107] Habib M, Ghosh N N, Sarkar R, Pramanik A, Sarkar P and Pal S 2018 Chem. Phys. Lett. 70921

[108] Saha S, Sarkar S, Pal S and Sarkar P 2013 J. Phys. Chem. C 11715890

[109] Lin C Y, Lai Y H, Chen H W, Chen J G, Kung C W, Vittal R et al 2011 Energy Environ. Sci. 43448

[110] Clarke T M and Durrant J R 2010 Chem. Rev. 1106736

[111] Sarkar S, Saha S, Pal S and Sarkar P 2014 RSC Adv. 414673
[112] Sarkar S, Rajbanshi B and Sarkar P 2014 J. Appl. Phys. 116 114303

[113] Sariciftci N S, Smilowitz L, Heeger A J and Wudl F 1992 Science 2581474

[114] Xie Q, Perez-Cordero E and Echegoyen L 1992 J. Am. Chem. Soc. 1143978

[115] Hiroshi I, Kiyoshi H, Tsuyoshi A, Masanori A, Seiji T, Tadashi O et al 1996 Chem. Phys. Lett. 263545

[116] Shaheen S E, Brabec C J, Sariciftci N S, Padinger F, Fromherz T and Hummelen J C 2001 Appl. Phys. Lett. 78841

[117] Brabec C J, Winder C, Sariciftci N S, Hummelen J C, Dhanabalan A, van Hal P A et al 2002 Adv. Funct. Mater. 12 709

[118] Halls J, Cornil J, Dos Santos D, Silbey R, Hwang D H, Holmes A et al 1999 Phys. Rev. B 605721

[119] Lemaur V, Steel M, Beljonne D, Brédas J L and Cornil J 2005 J. Am. Chem. Soc. 1276077

[120] Brédas J L, Beljonne D, Coropceanu V and Cornil J 2004 Chem. Rev. 1044971

[121] Joseph S, Ravva M K and Bredas J L 2017 J. Phys. Chem. Lett. 85171

[122] Li Y, Feng Y and Sun M 2015 Sci. Rep. 513970

[123] Song P, Li Y, Ma F and Sun M 2015 J. Mater. Chem. C 34810

[124] Li Y, Pullerits T, Zhao M and Sun M 2011 J. Phys. Chem. C 11521865

[125] Biswas S, Pramanik A and Sarkar P 2018 J. Phys. Chem. C 12214296

[126] Marcus R A and Sutin N 1985 Biochim. Biophys. Acta: Rev. Bioenerg. 811265

[127] Marcus R A 1993 Angew. Chem. Int. Ed. 321111

[128] Endres R, Fong C, Yang L, Witte G and Wöll C 2004 Comput. Mater. Sci. 29362

[129] Holmes D, Kumaraswamy S, Matzger A J and Vollhardt K P C 1999 Chem. A Eur. J. 53399

[130] Kaur I, Jia W, Kopreski R P, Selvarasah S, Dokmeci M R, Pramanik C et al 2008 J. Am. Chem. Soc. 13016274

[131] Park S, Kim S, Kim J, Whang C and Im S 2002 Appl. Phys. Lett. 802872

[132] Capozzi B, Dell E J, Berkelbach T C, Reichman D R, Venkataraman L and Campos L M 2014 J. Am. Chem. Soc. 13610486

[133] Brandbyge M, Mozos J L, Ordejón P, Taylor J and Stokbro K 2002 Phys. Rev. B 65165401

[134] Datta S 1997 Electronic transport in mesoscopic systems (Cambridge: Cambridge University Press)

[135] Gutiérrez R, Fagas G, Richter K, Grossmann F and Schmidt R 2003 Europhys. Lett. 6290

[136] Benesch C, Ćížek M, Thoss M and Domcke W 2006 Chem. Phys. Lett. 430355

[137] Xue Y and Ratner M A 2003 Phys. Rev. B 68 115406

[138] Neto A C, Guinea F, Peres N M, Novoselov K S and Geim A K 2009 Rev. Mod. Phys. 81109

[139] Manna A K and Pati S K 2013 J. Mater. Chem. C 13439

[140] Ren H, Li Q X, Luo Y and Yang J 2009 Appl. Phys. Lett. 94 173110

[141] Wang M and Li C M 2011 Phys. Chem. Chem. Phys. 135945

[142] Cheraghchi H and Esfarjani K 2008 Phys. Rev. B 78085123

[143] Son Y W, Cohen M L and Louie S G 2007 Nature 446342

[144] Hod O, Barone V and Scuseria G E 2008 Phys. Rev. B 77 035411 
[145] Kan E J, Li Z, Yang J and Hou J 2008 J. Am. Chem. Soc. 130 4224

[146] Lee Y L, Kim S, Park C, Ihm J and Son Y W 2010 ACS Nano 41345

[147] Yuan S, Dai C, Weng J, Mei Q, Ling Q, Wang L et al 2011 J. Phys. Chem. A $\mathbf{1 1 5} 4535$

[148] Bürgi T 2015 Nanoscale 715553

[149] Pakiari A and Jamshidi Z 2010 J. Phys. Chem. A 1149212

[150] Stokbro K, Taylor J, Brandbyge M, Mozos J L and Ordejon P 2003 Comput. Mater. Sci. 27151

[151] Manrique D Z, Al-Galiby Q, Hong W and Lambert C J 2016 Nano Lett. 161308

[152] Cheng Z L, Skouta R, Vazquez H, Widawsky J, Schneebeli S, Chen W et al 2011 Nat. Nanotechnol. 6353

[153] Chen W, Widawsky J R, Vázquez H, Schneebeli S T, Hybertsen M S, Breslow R et al 2011 J. Am. Chem. Soc. 13317160

[154] Ma G, Shen X, Sun L, Zhang R, Wei P, Sanvito S et al 2010 Nanotechnology 21495202

[155] Zang Y, Pinkard A, Liu Z F, Neaton J B, Steigerwald M L, Roy X et al 2017 J. Am. Chem. Soc. 13914845

[156] Clark M L, Rudshteyn B, Ge A, Chabolla S A, Machan C W, Psciuk B T et al 2016 J. Phys. Chem. C 1201657

[157] Roy P, Biswas S, Pramanik A and Sarkar P 2018 Chem. Phys. Lett. 70887

[158] Steck C, Franckevičius M, Zakeeruddin S M, Mishra A, Bäuerle P and Grätzel M 2015 J. Mater. Chem. A 317738

[159] Bi D, Mishra A, Gao P, Franckevičius M, Steck C, Zakeeruddin S M et al 2016 ChemSusChem 9433

[160] Taylor J, Guo H and Wang J 2001 Phys. Rev. B 63245407

[161] Ye Q and Chi C 2014 Chem. Mater. 264046

[162] Srinivas K, Yesudas K, Bhanuprakash K and Giribabu L 2009 J. Phys. Chem. C 11320117

[163] Zhou N, Prabakaran K, Lee B, Chang S H, Harutyunyan B, Guo P et al 2015 J. Am. Chem. Soc. 1374414

[164] Tarsang R, Promarak V, Sudyoadsuk T, Namuangruk S, Kungwan N, Khongpracha P et al 2015 RSC Adv. 538130

[165] Habib M, Saha S, Sarkar R, Pramanik A, Sarkar P and Pal S 2018 Comput. Theor. Chem. 113610

[166] Wenger S, Bouit P A, Chen Q, Teuscher J, Censo D D, Humphry-Baker R et al 2010 J. Am. Chem. Soc. 1325164

[167] Bouit P A, Villegas C, Delgado J L, Viruela P M, PouAmérigo R, Ortì̀a E et al 2011 Org. Lett. 13604

[168] Ogi D, Fujita Y, Mori S, Shirahata T and Misaki Y 2016 Org. Lett. 185868

[169] Feng J, Jiao Y, Ma W, Nazeeruddin M K, Grätzel M and Meng S 2013 J. Phys. Chem. C 1173772
[170] Scharber M C, Mühlbacher D, Koppe M, Denk P, Waldauf C, Heeger A J et al 2006 Adv. Mater. 18789

[171] Ghosh N N, Chakraborty A, Pal S, Pramanik A and Sarkar P 2014 Phys. Chem. Chem. Phys. 1625280

[172] Ghosh N N, Habib M, Pramanik A, Sarkar P and Pal S 2018 Bull. Mater. Sci. 4156

[173] Ryno S M, Ravva M K, Chen X, Li H and Brédas J L 2017 Adv. Energy Mater. 71601370

[174] Brédas J L, Norton J E, Cornil J and Coropceanu V 2009 Acc. Chem. Res. 421691

[175] Burke T M, Sweetnam S, Vandewal K and McGehee M D 2015 Adv. Energy Mater. 51500123

[176] Rozzi C A, Falke S M, Spallanzani N, Rubio A, Molinari E, Brida D et al 2013 Nat. Commun. 41602

[177] Gélinas S, Rao A, Kumar A, Smith S L, Chin A W, Clark J et al 2014 Science 343512

[178] Song Y, Clafton S N, Pensack R D, Kee T W and Scholes G D 2014 Nat. Commun. 54933

[179] Menke S M, Sadhanala A, Nikolka M, Ran N A, Ravva M K, Abdel-Azeim S et al 2016 ACS Nano 1010736

[180] Fukazawa A and Yamaguchi S 2009 Chem. Asian J. 4 1386

[181] Beenken W J and Pullerits T 2004 J. Phys. Chem. B 1086164

[182] Lu T and Chen F 2012 J. Mol. Graph. Model. 38314

[183] Zhang X, Chi L, Ji S, Wu Y, Song P, Han K et al 2009 J. Am. Chem. Soc. 13117452

[184] Kavarnos G J and Turro N J 1986 Chem. Rev. 86401

[185] Emelianova E, Van der Auweraer M and Bässler H 2008 J. Chem. Phys. 128224709

[186] Song P, Li Y, Ma F, Pullerits T and Sun M 2013 J. Phys. Chem. C 11715879

[187] Song P, Li Y, Ma F, Pullerits T and Sun M 2016 Chem. Rec. 16734

[188] D'Souza F, Chitta R, Ohkubo K, Tasior M, Subbaiyan N K, Zandler M E et al 2008 J. Am. Chem. Soc. 13014263

[189] Voityuk A A 2006 J. Chem. Phys. 124064505

[190] Tong M, Sheng C X and Vardeny Z V 2007 Phys. Rev. B 75 125207

[191] Mukamel S, Tretiak S, Wagersreiter T and Chernyak V 1997 Science 277781

[192] Zhong Y, Trinh M T, Chen R, Wang W, Khlyabich P P, Kumar B et al 2014 J. Am. Chem. Soc. 13615215

[193] Akimov A V and Prezhdo O V 2014 J. Am. Chem. Soc. 136 1599

[194] Pal S, Trivedi D J, Akimov A V, Aradi B, Frauenheim T and Prezhdo O V 2016 J. Chem. Theor. Comput. 121436 